\title{
Nearest-Neighbor Searching Under Uncertainty II
}

\author{
PANKAJ K. AGARWAL, Duke University \\ BORIS ARONOV, New York University \\ SARIEL HAR-PELED, University of Illinois at Urbana-Champaign \\ JEFF M. PHILLIPS, University of Utah \\ KE YI, Hong Kong University of Science and Technology \\ WUZHOU ZHANG, Apple Inc.
}

\begin{abstract}
Nearest-neighbor search, which returns the nearest neighbor of a query point in a set of points, is an important and widely studied problem in many fields, and it has a wide range of applications. In many of them, such as sensor databases, location-based services, face recognition, and mobile data, the location of data is imprecise. We therefore study nearest-neighbor queries in a probabilistic framework in which the location of each input point is specified as a probability distribution function. We present efficient algorithms for (i) computing all points that are nearest neighbors of a query point with nonzero probability and (ii) estimating the probability of a point being the nearest neighbor of a query point, either exactly or within a specified additive error.
\end{abstract}

Categories and Subject Descriptors: F.2 [Analysis of Algorithms and Problem Complexity]: Nonnumerical Algorithms and Problems; H.3.1 [Information Storage and Retrieval]: Content Analysis and Indexing-Indexing methods

General Terms: Algorithms, Theory

Additional Key Words and Phrases: Indexing uncertain data, probabilistic nearest neighbor, approximate nearest neighbor, threshold queries

ACM Reference Format:

Pankaj K. Agarwal, Boris Aronov, Sariel Har-Peled, Jeff M. Phillips, Ke Yi, and Wuzhou Zhang. 2016. Nearest-neighbor searching under uncertainty II. ACM Trans. Algorithms 13, 1, Article 3 (October 2016), 25 pages.

DOI: http://dx.doi.org/10.1145/2955098

A preliminary version of this article appeared in Proceedings of the ACM Symposium on Principles of Database Systems (PODS), 2013. The title Nearest-Neighbor Searching Under Uncertainty I has been reserved for the journal version of Agarwal et al. [2012].

Most of the work on this article was done while W. Zhang was at Duke University. P. K. Agarwal and W. Zhang were supported by the NSF under grants CCF-09-40671, CCF-10-12254, CCF-11-61359, and IIS-14-08846. B. Aronov was supported by NSF grants CCF-08-30691, CCF-11-17336, and CCF-12-18791, and by NSA MSP Grant H98230-10-1-0210. S. Har-Peled was supported by NSF grants CCF-09-15984 and CCF-1217462. J. Phillips was supported by NSF CCF-1350888, IIS-1251019, and ACI-1443046. K. Yi was supported by HKRGC under grants GRF-621413, GRF-16211614, and GRF-16200415.

Authors' addresses: P. K. Agarwal, Department of Computer Science, Box 90129, Duke University, Durham, NC 27708, USA; email: pankaj@cs.duke.edu; B. Aronov, Department of Computer Science and Engineering, Tandon School of Engineering, New York University, Brooklyn, New York 11201; email: boris.aronov@ nyu.edu; S. Har-Peled, Department of Computer Science, University of Illinois, 201 N. Goodwin Avenue; Urbana, IL 61801, USA; email: sariel@illinois.edu; J. M. Phillips, School of Computing, University of Utah, 50 S. Central Campus Dr. Salt Lake City, UT 84108, USA; email: jeffp@cs.utah.edu; K. Yi, Department of Computer Science and Engineering, Hong Kong University of Science and Technology, Clear Water Bay, Hong Kong, China; email: yike@ust.hk; W. Zhang, Apple Inc. 1 Infinite Loop, Cupertino, CA 95014, USA; email:wzhang@cs.duke.edu.

Permission to make digital or hard copies of part or all of this work for personal or classroom use is granted without fee provided that copies are not made or distributed for profit or commercial advantage and that copies show this notice on the first page or initial screen of a display along with the full citation. Copyrights for components of this work owned by others than ACM must be honored. Abstracting with credit is permitted. To copy otherwise, to republish, to post on servers, to redistribute to lists, or to use any component of this work in other works requires prior specific permission and/or a fee. Permissions may be requested from Publications Dept., ACM, Inc., 2 Penn Plaza, Suite 701, New York, NY 10121-0701 USA, fax +1 (212) 869-0481, or permissions@acm.org.

(c) 2016 ACM 1549-6325/2016/10-ART3 $\$ 15.00$

DOI: http://dx.doi.org/10.1145/2955098 


\section{INTRODUCTION}

Nearest-neighbor search is a fundamental problem in data management. It has applications in such diverse areas as spatial databases, information retrieval, data mining, pattern recognition, and so forth. In its simplest form, it asks for preprocessing a set $S$ of $n$ points in $\mathbb{R}^{d}$ into a data structure so that given any query point $q$, the nearest neighbor (NN) of $q$ in $S$ can be reported efficiently. This problem has been studied extensively in database, machine-learning, and computational geometry communities, and is now relatively well understood. However, in some of the applications mentioned previously, data are imprecise and are often modeled as probabilistic distributions. This has led to a flurry of research activities on query processing over probabilistic data, including the NN problem; see Aggarwal [2009] and Dalvi et al. [2009] for surveys on uncertain data, and see, for example, Cheng et al. [2010] and Ljosa and Singh [2007] for application scenarios of NN search under uncertainty.

Despite many efforts devoted to the probabilistic NN problem, it still lacks a theoretical foundation. Specifically, not only have we yet to understand its complexity (is the problem inherently more difficult than on precise data?), but also we lack efficient algorithms to solve it. Furthermore, existing solutions all use heuristics without nontrivial performance guarantees. This article addresses some of these issues.

\subsection{Problem Definition}

An uncertain point $P$ in $\mathbb{R}^{2}$ is represented as a continuous probability distribution defined by a probability density function (pdf) $f_{P}: \mathbb{R}^{2} \rightarrow \mathbb{R}_{\geq 0} ; f_{P}$ may be a parametric pdf such as a uniform distribution or a Gaussian distribution, or may be a nonparametric pdf such as a histogram. ${ }^{1}$ The uncertainty region of $P$ (or the support of $f_{P}$ ) is the set of points for which $f_{P}$ is positive, that is, $\operatorname{Sup} f_{P}=\left\{x \in \mathbb{R}^{2} \mid f_{P}(x)>0\right\}$. We assume $P$ has a bounded uncertainty region: if $f_{P}$ is Gaussian, we work with the truncated Gaussian, as in Beskales et al. [2008] and Cheng et al. [2008]. We also consider the case where $P$ is represented as a discrete distribution defined by a finite set $P=\left\{p_{1}, \ldots, p_{k}\right\} \subset \mathbb{R}^{2}$ along with a set of location probabilities $\left\{w_{1}, \ldots, w_{k}\right\} \subset(0,1]$, where $w_{i}=\operatorname{Pr}\left[P\right.$ is $\left.p_{i}\right]$ and $\sum_{i=1}^{k} w_{i}=1$; and we say that $P$ has a discrete distribution of description complexity $k$. Let $\mathcal{P}=\left\{P_{1}, \ldots, P_{n}\right\}$ be a set of $n$ uncertain points in $\mathbb{R}^{2}$, and let $d(\cdot, \cdot)$ be the Euclidean distance.

Fix a point $q \in \mathbb{R}^{2}$ and an integer $i \in\{1, \ldots, n\}$. We define $\pi_{i}(q)=\pi\left(P_{i}, q\right)$ to be the probability of $P_{i} \in \mathcal{P}$ being the nearest neighbor of $q$, referred to as the quantification probability of $q$ (for $P_{i}$ ). Next, let $g_{q, i}$ be the pdf of the distance between $q$ and $P_{i}$. That is,

$$
g_{q, i}(r)=\operatorname{Pr}\left[r \leq d\left(q, P_{i}\right) \leq r+\mathrm{d} r\right] / \mathrm{d} r .
$$

See Figure 1 for an example of $g_{q, i}$. Let $G_{q, i}(r)=\int_{0}^{r} g_{q, i}\left(r^{\prime}\right) \mathrm{d} r^{\prime}$ denote the cumulative distribution function (cdf) of the distance between $q$ and $P_{i}$. Note that if $P_{i}$ is the NN of $q$ and $d\left(P_{i}, q\right)=r$, then $d\left(P_{j}, q\right)>r$ for all $j \neq i$. Therefore, $\pi_{i}(q)$ can be expressed as follows:

$$
\pi_{i}(q)=\int_{0}^{\infty} g_{q, i}(r) \prod_{j \neq i}\left(1-G_{q, j}(r)\right) \mathrm{d} r
$$

\footnotetext{
${ }^{1}$ If the location of data is precise, we call it certain. The probabilistic model we use is often called the locational model, where the location of an uncertain point follows the given distribution. This is to be contrasted with the existential model, where each point has a precise location but it appears with a given probability.
} 


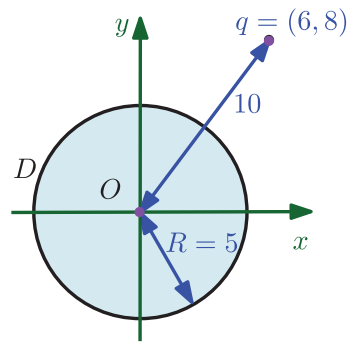

(a)

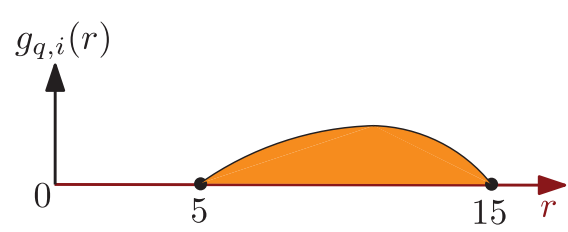

(b)

Fig. 1. (a) $P_{i}$ is represented by a uniform distribution defined on a disk $D$ of radius $R=5$ and centered at origin $O, q=(6,8)$. (b) $g_{q, i}(r)$, the pdf of the distance function between $q$ and $P_{i}$.

If $P_{i}$ s are represented by discrete distributions, then Equation (1) can be rewritten as follows:

$$
\pi_{i}(q)=\sum_{p_{i s} \in P_{i}} w_{i s} \prod_{j \neq i}\left(1-G_{q, j}\left(d\left(p_{i s}, q\right)\right)\right)
$$

where

$$
G_{q, j}(r)=\sum_{d\left(p_{j t}, q\right) \leq r} w_{j t} .
$$

Given a set $\mathcal{P}$ of $n$ uncertain points, the probabilistic nearest neighbor (PNN) problem is to preprocess $\mathcal{P}$ into a data structure so that, for any given query point $q$, we can efficiently return all pairs $\left(P_{i}, \pi_{i}(q)\right)$ with $\pi_{i}(q)>0$.

Usually, the PNN problem is divided into the following two subproblems, which are often considered separately.

Nonzero NNs. The first subproblem is to find all the $P_{i}$ s with $\pi_{i}(q)>0$ without computing the actual quantification probabilities, that is, to find

$$
\mathrm{NN}_{\neq 0}(q, \mathcal{P})=\left\{P_{i} \mid \pi_{i}(q)>0\right\} .
$$

If the point set $\mathcal{P}$ is obvious from the context, we drop the argument $\mathcal{P}$ from $\mathrm{NN}_{\neq 0}(q, \mathcal{P})$ and write it as $\mathrm{NN}_{\neq 0}(q)$. Note that $\mathrm{NN}_{\neq 0}(q)$ depends (besides $q$ ) only on the uncertainty regions of the uncertain points, but not on the actual pdf's.

A possible approach to compute nearest neighbors is to use Voronoi diagrams. For example, the standard Voronoi diagram of a set of certain points in $\mathbb{R}^{2}$ is the planar subdivision so that all points in the same face have the same nearest neighbor. In our case, we define the nonzero Voronoi diagram, denoted by $\mathcal{V}_{\neq 0}(\mathcal{P})$, to be the subdivision of $\mathbb{R}^{2}$ into maximal connected regions such that $\mathrm{NN}_{\neq 0}(q)$ is the same for all points $q$ within each region. That is, for a subset $\mathcal{T} \subseteq \mathcal{P}$, let

$$
\operatorname{cell}_{\neq 0}(\mathcal{T})=\left\{q \in \mathbb{R}^{2} \mid \mathrm{NN}_{\neq 0}(q)=\mathcal{T}\right\} .
$$

Although there are $2^{n}$ subsets of $\mathcal{P}$, we will see later that only a small number of them have nonempty Voronoi cells. The planar subdivision $\mathcal{V}_{\neq 0}(\mathcal{P})$ is induced by all the nonempty cell $\neq 0(\mathcal{T})_{\mathrm{s}}$ for $\mathcal{T} \subseteq \mathcal{P}$. The (combinatorial) complexity of $\mathcal{V}_{\neq 0}(\mathcal{P})$ is the total number of vertices, edges, and faces in $\mathcal{V}_{\neq 0}(\mathcal{P})$. The complexity of the Voronoi diagram is often regarded as a measure of the complexity of the corresponding nearest-neighbor problem. 
In this article, we study the worst-case complexity of $\nu_{\neq 0}(\mathcal{P})$ and how it can be efficiently constructed. In addition, once we have $\mathcal{V}_{\neq 0}(\mathcal{P})$, it can be preprocessed into a point-location structure to support $\mathrm{NN}_{\neq 0}$ queries in logarithmic time.

Computing Quantification Probabilities. The second subproblem is to compute the quantification probability $\pi_{i}(q)$ for a given $q$ and $P_{i}$. Exact values of these probabilities are often unstable - a far-away point can affect these probabilities-and computing them requires complex $n$-dimensional integration (see Equation (1)), which is often expensive. As such, we resort to computing $\pi_{i}(q)$ approximately within a given additive error tolerance $\varepsilon \in(0,1)$. More precisely, we aim at returning a value $\widehat{\pi}_{i}(q)$ such that $\left|\pi_{i}(q)-\widehat{\pi}_{i}(q)\right| \leq \varepsilon$.

\subsection{Previous Work}

Nonzero NNs. Sember and Evans [2008] showed that if the uncertainty regions of the points in $\mathcal{P}$ are disks, then the complexity of $\mathcal{V}_{\neq 0}(\mathcal{P})$ is $O\left(n^{4}\right)$ (though they did not use this term explicitly); they did not offer any lower bound. If one only considers those cells of $\mathcal{V}_{\neq 0}(\mathcal{P})$ in which $\mathrm{NN}_{\neq 0}(q)$ contains only one uncertain point $P_{i}$ (i.e., only $P_{i}$ has a nonzero probability of being the $\mathrm{NN}$ of $q$ ), they showed that the complexity of these cells is $O(n)$. Note that for such a cell, we always have $\pi_{i}(q)=1$ for any $q$ in the cell, so they form the guaranteed Voronoi diagram. Probably unaware of the work by Sember and Evans [2008] and Cheng et al. [2010] proved an exponential upper bound for the complexity of the nonzero Voronoi diagram, which they referred to as the UV-diagram.

The nonzero Voronoi diagram is not the only way to find the nonzero NN s. Cheng et al. [2004] designed a branch-and-prune solution based on the $R$-tree. Recently, Zhang et al. [2013] proposed to combine the nonzero Voronoi diagram with R-tree-like bounding rectangles. These methods do not provide any nontrivial performance guarantees.

Computing Quantification Probabilities. Computing the quantification probabilities has attracted much attention in the database community. Cheng et al. [2004] used numerical integration, which is quite expensive. Cheng et al. [2008] and Bernecker et al. [2011] proposed some filter refinement methods to give upper and lower bounds on the quantification probabilities. Kriegel et al. [2007] took a random sample from the continuous distribution of each uncertain point to convert it to a discrete one, so that the integration becomes a sum, and they clustered each sample to further reduce the complexity of the query computation. Dai et al. [2005] considered the problem of reporting points $P_{i}$ for which $\pi_{i}(q)$ exceeds some given threshold. We note that these methods are best-effort based: they do not always give the $\varepsilon$-error that we aim at-how tight the resulting bounds are depends on the data.

Other Variants of the Problem. The PNN problem we focus on in this article is the most commonly studied version of the problem, but many variants and extensions have been considered.

Besides using the quantification probability, one can also consider the expected distance from a query point $q$ to an uncertain point and return the one minimizing the expected distance as the nearest neighbor; this was studied by Agarwal et al. [2012]. This NN definition is easier since the expected distance to each uncertain point can be computed separately, whereas the quantification probability involves the interaction among all uncertain points. However, the expected nearest neighbor is not a good indicator under large uncertainty (see Yuen et al. [2010] for details).

Instead of returning only the nearest neighbor, one can ask to return the $k$ nearest neighbors in a ranked order (the $k N N$ problem). If we use expected distance, the ranking of points is straightforward, namely, rank them in a nondecreasing order of the expected distance from the query point. However, when quantification probabilities 
are considered, many different criteria for ranking the results are possible, leading to different problem variants [Jestes et al. 2011].

Various combinations of these extensions have been studied in the literature (see, e.g., Beskales et al. [2008], Cheng et al. [2009], Kamousi et al. [2014], Ljosa and Singh [2007], and Yuen et al. [2010]).

\subsection{Our Results}

The main results of this article are the following:

(i) $\mathrm{A} \Theta\left(n^{3}\right)$ bound on the combinatorial complexity $\mathcal{V}_{\neq 0}(\mathcal{P})$, an improved quadratic bound on the complexity of $\mathcal{V}_{\neq 0}(\mathcal{P})$ for a special case, and an efficient randomized algorithm for computing $\mathcal{V}_{\neq 0}(\mathcal{P})$

(ii) Near-linear-size data structures for answering $\mathrm{NN}_{\neq 0}$ queries in polylogarithmic or sublinear time

(iii) Efficient data structures for computing the quantification probabilities of a query point approximately.

We now describe these results in more detail:

Nonzero Voronoi Diagrams. We first study (in Section 2) the complexity of $\mathcal{V}_{\neq 0}(\mathcal{P})$. Suppose the uncertainty region of each $P_{i} \in \mathcal{P}$ is a disk and $d(\cdot, \cdot)$ is the $L_{2}$ metric. We show that $\mathcal{V}_{\neq 0}(\mathcal{P})$ has $O\left(n^{3}\right)$ complexity, and that this bound is tight in the worst case even if all uncertainty-region disks have the same radius. This significantly improves the bound in Sember and Evans [2008] and closes the problem. We also show that the $O\left(n^{3}\right)$ bound holds for a much larger class of uncertainty regions, namely, even if each uncertainty region is a semialgebraic set of constant description complexity; see Section 2 for the definition of a semialgebraic set.

If the disks are pairwise disjoint and the ratio of their radii is at most $\lambda$, then the complexity of $\mathcal{V}_{\neq 0}(\mathcal{P})$ is $O\left(\lambda n^{2}\right)$, and we prove a lower bound of $\Omega\left(n^{2}\right)$. Again, this bound holds for a larger class of uncertainty regions.

We show that if each point in $\mathcal{P}$ has a discrete distribution of description complexity at most $k$, then $\mathcal{V}_{\neq 0}(\mathcal{P})$ has $O\left(k n^{3}\right)$ complexity.

We present a randomized, output-sensitive algorithm for computing $\nu_{\neq 0}(\mathcal{P})$ in $O\left(n^{2} \log n+\mu\right)$ expected time, where $\mu$ is the complexity of $\mathcal{V}_{\neq 0}(\mathcal{P})$. $\mathcal{P}$ can be preprocessed into a point-location structure of size $O(\mu)$ that can answer an $\mathrm{NN}_{\neq 0}$ query in $O(\log n+t)$ time, where $t$ is the output size [de Berg et al. 2008].

Answering $N N_{\neq 0}$ Queries. Since the complexity of $\mathcal{V}_{\neq 0}(\mathcal{P})$ can be cubic in the worst case, in Section 3 we present near-linear-size data structures for answering $\mathrm{NN}_{\neq 0}$ queries efficiently. In particular, if the uncertainty region of each point is a disk, then an $\mathrm{NN}_{\neq 0}$ query can be answered in $O(\log n+t)$ time using $O(n$ polylog $(n))$ space, where $t$ is the output size. If each point of $\mathcal{P}$ has a discrete distribution of size at most $k$, then an $\mathrm{NN}_{\neq 0}$ query can be answered in $O\left(N^{1 / 2} \log ^{3} N+t\right)$ time using $O\left(N \log ^{2} N\right)$ space, where $t$ is the output size and $N=n k$. These results rely on geometric data structures for answering simplex range queries and their variants. See Agarwal [2016] for a recent survey.

Computing Quantification Probabilities. Next, in Section 4, we focus our attention on computing quantification probabilities for a query point $q$, that is, reporting the values of $\pi_{i}(q)$ for all $P_{i}$ s for which $\pi_{i}(q)>0$. We begin in Section 4.1 by describing a data structure that can compute quantification probabilities exactly if each $P_{i}$ has a discrete distribution of size at most $k$. Its size is $O\left(N^{4}\right)$ and it can return all $t$ positive quantification probabilities for a query point in time $O(\log N+t)$, where $N=n k$ as 
noted earlier. Since computing quantification probabilities is expensive even for points with discrete distributions, we mostly focus on computing them approximately.

We present two data structures for approximating the quantification probabilities efficiently. The first (see Section 4.2) is a Monte-Carlo algorithm for estimating $\pi_{i}(q)$ for any $P_{i}$ and $q$ within additive error $\varepsilon$ with probability at least $1-\delta$, for parameters $\varepsilon, \delta \in(0,1)$. We argue that if each uncertain point has a discrete distribution of size at most $k$, then we can estimate $\pi_{i}(q)$ within additive error $\varepsilon$ with probability at least $1-\delta$ by using $s_{\varepsilon, \delta}=O\left(\left(1 / \varepsilon^{2}\right) \log (N / \delta)\right)$ random instantiations of $\mathcal{P}$. (Note that there are at most $1 / \varepsilon P_{i}$ s for which $\pi_{i}(q)>\varepsilon$.) Consequently, we can preprocess $\mathcal{P}$ into a data structure of size $O\left(\left(n / \varepsilon^{2}\right) \log (N / \delta)\right)$ so that for any query point $q \in \mathbb{R}^{2}, \pi_{i}(q)$ for all $P_{i}$ s can be estimated within additive error $\varepsilon$ in $O\left(\left(1 / \varepsilon^{2}\right) \log (N / \delta) \log n\right)$ time, with probability at least $1-\delta$. The algorithm explicitly computes the estimates of $\pi_{i}(q) \mathrm{s}$ for at most $s_{\varepsilon, \delta}$ points and sets the estimate to 0 for the rest of the points. We also show that this approach works even if the distribution of each $P_{i}$ is continuous, by approximating a continuous distribution with a discrete one. A key observation is that it suffices to sample a polynomial number of points from the distribution of each $P_{i}$ to ensure that the error in the quantification probability is at most $\varepsilon$.

Next, in Section 4.3, we describe a deterministic algorithm for computing $\pi_{i}(q)$ approximately if each point has a discrete distribution of size at most $k$. We show that $\mathcal{P}$ can be preprocessed into a data structure of $O(N)$ size so that for any $q \in \mathbb{R}^{2}$ and for any $\varepsilon \in(0,1), \pi_{i}(q)$, for all $i \in\{1, \ldots, n\}$, can be computed with additive error at most $\varepsilon$ in $O(\rho k \log (\rho / \varepsilon)+\log N)$ time, where $\rho$ is the ratio of the largest to the smallest location probabilities over all possible locations of points in $\mathcal{P}$. We show that there are at most $m(\rho, \varepsilon)=\rho k \ln (\rho / \varepsilon)+k-1$ points of $\mathcal{P}$ for which $\pi_{i}(q)>\varepsilon$. The algorithm explicitly estimates $\pi_{i}(q)$ for at most $m(\rho, \varepsilon)$ points and sets the estimate to 0 for the rest of the points.

\section{NONZERO PROBABILISTIC VORONOI DIAGRAM}

Let $\mathcal{P}$ be a set of $n$ uncertain points as described earlier. We analyze the combinatorial structure of $\mathcal{V}_{\neq 0}(\mathcal{P})$ and describe algorithms for constructing it. We first consider the case when the distribution of each point is continuous and then consider the discrete case.

\subsection{Continuous Case}

For simplicity, we first assume that the uncertainty region of each $P_{i}$ is a circular disk $D_{i}$ of radius $r_{i}$ centered at $c_{i}$.

We first observe that the structure of $\mathcal{V}_{\neq 0}(\mathcal{P})$ does not depend on the actual pdf of $P_{i}$ s. What really matters is the uncertainty region $D_{i}$. More precisely, for each $1 \leq i \leq n$ and for $q \in \mathbb{R}^{2}$, let

$$
\begin{aligned}
& \Delta_{i}(q)=\max _{p \in D_{i}} d(q, p)=d\left(q, c_{i}\right)+r_{i}, \\
& \delta_{i}(q)=\min _{p \in D_{i}} d(q, p)=\max \left\{d\left(q, c_{i}\right)-r_{i}, 0\right\}
\end{aligned}
$$

be the maximum and minimum possible distance, respectively, from $q$ to $P_{i}$.

The following lemma, whose proof is straightforward, characterizes the structure of $V_{\neq 0}(\mathcal{P})$.

Lemma 2.1. For a point $q \in \mathbb{R}^{2}$, a point $P_{i} \in \mathcal{P}$ belongs to $N N_{\neq 0}(q, \mathcal{P})$ if and only if

$$
\delta_{i}(q)<\Delta_{j}(q) \text { for all } 1 \leq j \neq i \leq n .
$$



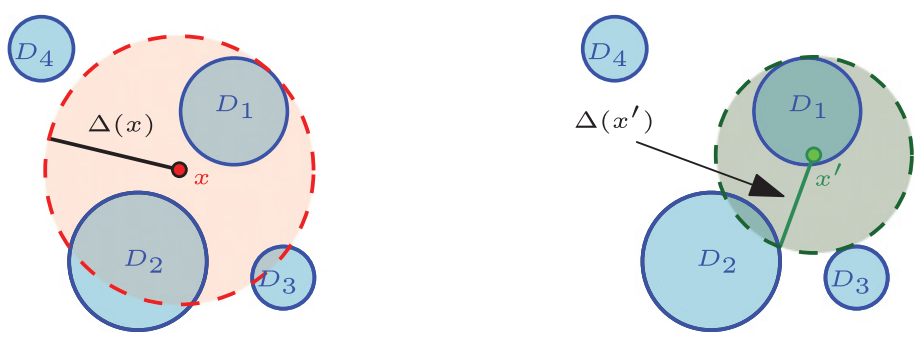

Fig. 2. $\mathcal{P}=\left\{P_{1}, \ldots, P_{5}\right\}, \Delta(x)=\Delta_{1}(x), \mathrm{NN}_{\neq 0}(x, \mathcal{P})=\left\{P_{1}, P_{2}, P_{3}\right\}, \Delta\left(x^{\prime}\right)=\Delta_{1}\left(x^{\prime}\right), \mathrm{NN}_{\neq 0}\left(x^{\prime}, \mathcal{P}\right)=\left\{P_{1}, P_{2}\right\}$, and $x^{\prime}$ lies on an edge of $\mathcal{V}_{\neq 0}(\mathcal{P})$.

Let $\Delta: \mathbb{R}^{2} \rightarrow \mathbb{R}$ denote the lower envelope $e^{2}$ of $\Delta_{1}, \ldots, \Delta_{n}$; that is, for any $q \in \mathbb{R}^{2}$,

$$
\Delta(q)=\min _{1 \leq i \leq n} \Delta_{i}(q)
$$

The projection of the graph of $\Delta(x)$ onto the $x y$-plane is the additive-weighted Voronoi diagram of the points $c_{1}, \ldots, c_{n}$, where the weight of $c_{i}$ is $r_{i}$, and the weighted distance from $q$ to $c_{i}$ is $d\left(q, c_{i}\right)+r_{i}$, for $i=1, \ldots, n$. Let $\mathbb{M}$ denote this planar subdivision. It has linear complexity and each of its edges is a hyperbolic arc; see Ash and Bolker [1986]. Lemma 2.1 implies that, for any $q \in \mathbb{R}^{2}$,

$$
\mathrm{NN}_{\neq 0}(q, \mathcal{P})=\left\{P_{i} \mid \delta_{i}(q)<\Delta(q)\right\} .
$$

See Figure 2. It also implies that, as we move $x$ continuously in $\mathbb{R}^{2}, \mathrm{NN}_{\neq 0}(x, \mathcal{P})$ remains the same until $\delta_{i}(x)$, for some $1 \leq i \leq n$, becomes equal to $\Delta(x)$ (e.g., $x^{\prime}$ in Figure 2). This observation was made in earlier papers as well; see, for example, Cheng et al. [2008, 2004]. Using this observation, we can now characterize $\mathcal{V}_{\neq 0}(\mathcal{P})$.

For $i=1, \ldots, n$, let $\gamma_{i}=\left\{x \in \mathbb{R}^{2} \mid \delta_{i}(x)=\Delta(x)\right\}$ be the zero set of the function $\Delta(x)-\delta_{i}(x)$. Set $\Gamma=\left\{\gamma_{1}, \ldots, \gamma_{n}\right\}$.

The curve $\gamma_{i}$ partitions the plane into two open regions: $\Delta(x)<\delta_{i}(x)$ and $\Delta(x)>\delta_{i}(x)$. By Equation (4), $P_{i} \in \mathrm{NN}_{\neq 0}(x, \mathcal{P})$ for all points $x$ inside the latter region and for none of the points $x$ inside the former region. It is well known that, for any fixed $j \neq i$, $\gamma_{i j}=\left\{x \in \mathbb{R}^{2} \mid \delta_{i}(x)=\Delta_{j}(x)\right\}$ is a hyperbolic curve [Ash and Bolker 1986]. The curve $\gamma_{i}$ is composed of pieces of $\gamma_{i j}$, for $j \neq i$. We refer to the endpoints of these pieces as breakpoints of $\gamma_{i}$. They are the intersection points of $\gamma_{i}$ with an edge of $\mathbb{M}$ and correspond to points $q$ such that the disk of radius $\Delta(q)$ centered at $q$ touches (at least) two disks of $\mathcal{D}$ from inside, touches $D_{i}$ from outside, and does not contain any disk of $\mathcal{D}$ in its interior. See Figure 3. Formally, we say that a disk $D_{1}$ touches a disk $D_{2}$ from the outside (inside, respectively) if $\partial D_{1} \cap \partial D_{2} \neq \emptyset$ and $\operatorname{int} D_{1} \cap \operatorname{int} D_{2}=\emptyset\left(\operatorname{int} D_{2} \subseteq \operatorname{int} D_{1}\right.$, respectively).

Lemma 2.2. The curve $\gamma_{i}, 1 \leq i \leq n$, has at most $2 n$ breakpoints, and it can be computed in $O(n \log n)$ time.

Proof. Let $\Gamma_{i}=\left\{\gamma_{i j} \mid j \neq i, 1 \leq j \leq n\right\}$. It can be verified that a ray emanating from $c_{i}$ intersects the hyperbolic curve $\gamma_{i j}$, for any $j \neq i$, in at most one point, so $\gamma_{i j}$ can be viewed as the graph of a function in polar coordinates with $c_{i}$ as the origin. That is, let $\gamma_{i j}:[0,2 \pi) \rightarrow \mathbb{R}_{\geq 0}$, where $\gamma_{i j}(\theta)$ is the distance from $c_{i}$ to $\gamma_{i j}$ in direction $\theta$. Then, $\gamma_{i}$ is the lower envelope of $\Gamma_{i}$. Since each pair of curves in $\Gamma_{i}$ intersects at most twice, a well-known result on lower envelopes implies that $\gamma_{i}$ has at most $2 n$ breakpoints, and

${ }^{2}$ The lower envelope, $L_{F}$, of a set $F$ of functions is their pointwise minimum, that is, $L_{F}(x)=\min f \in F f(x)$. The upper envelope, $U_{F}$, of $F$ is the pointwise maximum, that is, $U_{F}(x)=\max _{f \in F} f(x)$. 


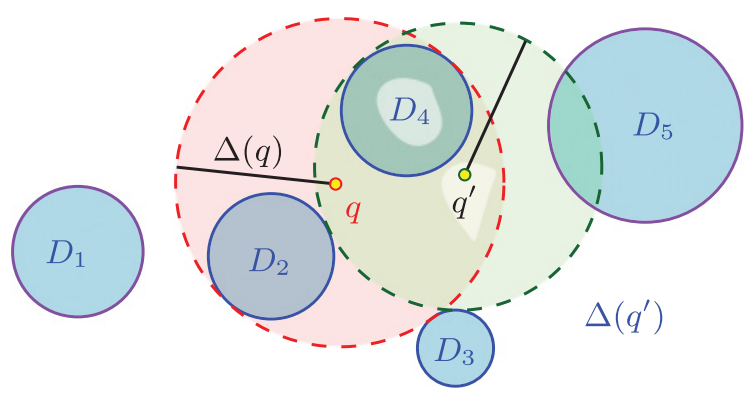

Fig. 3. The point $q$ is a break point of $\gamma_{3}$ and $q^{\prime}$ is an intersection point of $\gamma_{2}$ and $\gamma_{3}$.

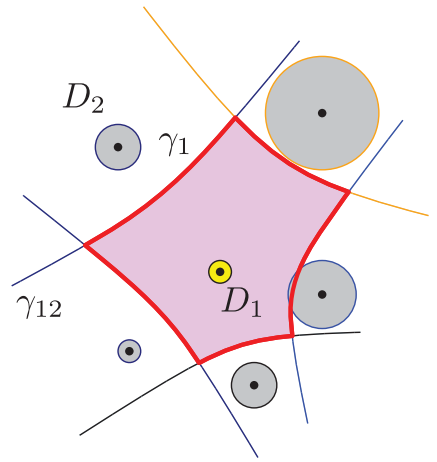

Fig. 4. An example of $\gamma_{1}$.

that it can be computed in $O(n \log n)$ time [Sharir and Agarwal 1995]. See Figure 4 for an example.

Let $\mathcal{A}(\Gamma)$ denote the planar subdivision induced by $\Gamma$ : its vertices are the breakpoints of $\gamma_{i} \mathrm{~s}$ and the intersection points of two curves in $\Gamma$, its edges are the portions of $\gamma_{i} \mathrm{~s}$ between two consecutive vertices, and its cells are the maximal connected regions of the plane that do not intersect any curve of $\Gamma$. We refer to vertices, edges, and cells of $\mathcal{A}(\Gamma)$ as its 0 -, 1-, and 2-dimensional faces.

For a face $\phi$ (of any dimension), and for any two points $x, y \in \phi$, the sets $\left\{P_{i} \mid \delta_{i}(x)<\right.$ $\Delta(x)\}$ and $\left\{P_{j} \mid \delta_{j}(y)<\Delta(y)\right\}$ are the same; we denote this set by $\mathcal{P}_{\phi}$. Furthermore, if $x, y$ lie in two neighboring faces $\phi$ and $\phi^{\prime}$, respectively, then $\mathcal{P}_{\phi} \neq \mathcal{P}_{\phi^{\prime}}$. The following lemma is an immediate consequence of Equation (4).

Lemma 2.3. For all points $x$ lying in a face $\phi$ of $\mathcal{A}(\Gamma), N N_{\neq 0}(x, \mathcal{P})=\mathcal{P}_{\phi}$.

For a subset $\mathcal{T} \subseteq \mathcal{P}$, let cell $\neq 0(\mathcal{T})$ be as defined in Equation (3). An immediate corollary of the previous lemma is:

Corollary 2.4. (i) For any $\mathcal{T} \subseteq \mathcal{P}$, $\operatorname{cell}_{\neq 0}(\mathcal{T}) \neq \emptyset$ if and only if there is a face $\phi$ of $\mathcal{A}(\Gamma)$ with $\mathcal{T}=\mathcal{P}_{\phi}$.

(ii) The planar subdivision $\mathcal{A}(\Gamma)$ coincides with $\mathcal{V}_{\neq 0}(\mathcal{P})$.

We now bound the complexity of $\mathcal{A}(\Gamma)$ and thus of $\mathcal{V}_{\neq 0}(\mathcal{P})$.

TheOREM 2.5. Let $\mathcal{P}=\left\{P_{1}, \ldots, P_{n}\right\}$ be a set of $n$ uncertain points in $\mathbb{R}^{2}$ whose uncertainty regions are disks. Then $\mathcal{V}_{\neq 0}(\mathcal{P})$ has $O\left(n^{3}\right)$ complexity. Moreover, it can be computed in $O\left(n^{2} \log n+\mu\right)$ expected time, where $\mu$ is the complexity of $\mathcal{V}_{\neq 0}(\mathcal{P})$. 
PRoof. Using a standard perturbation argument (see, e.g., Sharir and Agarwal [1995]), it suffices to bound the complexity of $\mathcal{V}_{\neq 0}(\mathcal{P})$ when the disks corresponding to the uncertainty regions of the points of $\mathcal{P}$ are in a general position, so we can assume that the degree of every vertex in $\mathcal{V}_{\neq 0}(\mathcal{P})$ is constant. Since $\mathcal{V}_{\neq 0}(\mathcal{P})$ is a planar subdivision and the degree of every vertex is constant, the number of edges and cells in $\mathcal{V}_{\neq 0}(\mathcal{P})$ is proportional to the number of its vertices. Hence, it suffices to bound the number of vertices. Let $\Gamma=\left\{\gamma_{1}, \ldots, \gamma_{n}\right\}$ be the set of curves as defined earlier. By Lemma 2.2, each $\gamma_{i}$ has $O(n)$ breakpoints, so there are a total of $O\left(n^{2}\right)$ breakpoints. We claim that each pair of curves $\gamma_{i}$ and $\gamma_{j}$ intersect $O(n)$ times-each such intersection point corresponds to a point $v \in \mathbb{R}^{2}$ such that the disk of radius $\Delta(v)$ centered at $v$ touches $D_{i}$ and $D_{j}$ from the outside and another disk $D_{k}$ of $\mathcal{D}$, the one realizing the value of $\Delta(v)$, from the inside (e.g., $q^{\prime}$ in Figure 3). For a fixed $k$, we show that there are at most two points $v$ such that $\delta_{i}(v)=\delta_{j}(v)=\Delta_{k}(v)$. Note that $\delta_{i}(v)=\Delta_{k}(v)$ represents either an empty set or one hyperbolic branch, and the same holds for $\delta_{j}(v)=\Delta_{k}(v)$. Two such hyperbolic branches intersect at most twice, implying that $\delta_{i}(v)=\delta_{j}(v)=\Delta_{k}(v)$ contributes at most two vertices. Hence, the number of vertices in $\mathcal{V}_{\neq 0}(\mathcal{P})$ is $O\left(n^{3}\right)$, as claimed.

By Lemma 2.2, $\Gamma$ can be computed in $O\left(n^{2} \log n\right)$ time. The planar subdivision $\mathcal{A}(\Gamma)$ can be computed in $O(n \log n+\mu)$ expected time using the randomized incremental method [Agarwal and Sharir 2000], where $\mu$ is the complexity of $\mathcal{V}_{\neq 0}(\mathcal{P})$. Hence, $\mathcal{V}_{\neq 0}(\mathcal{P})$ can be computed in $O\left(n^{2} \log n+\mu\right)$ expected time.

The previous argument is quite general and extends to a large class of uncertainty regions. In particular, a two-dimensional semialgebraic set is a subset of $\mathbb{R}^{2}$ obtained from a finite number of sets of the form $\left\{x \in \mathbb{R}^{2} \mid g(x) \geq 0\right\}$, where $g$ is a bivariate polynomial with real coefficients, by Boolean operations (union, intersection, and complement). A semialgebraic set has constant description complexity if the number of polynomials defining the set as well as the maximum degree of these polynomials is a constant. For example, a polygon with a constant number of edges and a region defined by a constant number of quadratic arcs are semialgebraic sets of constant description complexity.

Suppose the uncertainty region of each point in $\mathcal{P}$ is a semialgebraic set of constant description complexity, denoted by $\sigma_{i}$.

The analysis for the case of disks shows that a vertex of $\mathcal{V}_{\neq 0}(\mathcal{P})$ is the center of a disk that touches uncertainty regions of three different points. Fix a triple of uncertainty regions $\sigma_{i}, \sigma_{j}, \sigma_{k}$. Since they are semialgebraic sets of constant complexity, there are only $O(1)$ disks that are tangent to $\sigma_{1}, \sigma_{2}$, and $\sigma_{3}$ simultaneously. Therefore, $\mathcal{V}_{\neq 0}(\mathcal{P})$ has $O\left(n^{3}\right)$ vertices, which in view of the earlier discussion implies that $\mathcal{V}_{\neq 0}(\mathcal{P})$ has $O\left(n^{3}\right)$ combinatorial complexity. Assuming an extension of the real RAM model of computation in which the roots of polynomials of constant degree can be computed exactly in $O(1)$ time, the randomized algorithm described earlier can be extended to this case as well. Omitting further details, we conclude the following:

Theorem 2.6. Let $\mathcal{P}=\left\{P_{1}, \ldots, P_{n}\right\}$ be a set of $n$ uncertain points in $\mathbb{R}^{2}$ whose uncertainty regions are semialgebraic sets of constant description complexity. Then $\nu_{\neq 0}(\mathcal{P})$ has $O\left(n^{3}\right)$ complexity. Moreover, it can be computed in $O\left(n^{2} \log n+\mu\right)$ expected time, where $\mu$ is the complexity of $\mathcal{V}_{\neq 0}(\mathcal{P})$.

Next, we show that the maximum complexity of $\mathcal{V}_{\neq 0}(\mathcal{P})$ is $\Omega\left(n^{3}\right)$ even if the uncertainty regions of points in $\mathcal{D}$ are disks of the same radius.

THEOREM 2.7. There exists a set $\mathcal{P}$ of $n$ uncertain points, whose uncertainty regions are disks of the same radius, for which $\mathcal{V}_{\neq 0}(\mathcal{P})$ has $\Omega\left(n^{3}\right)$ vertices. 


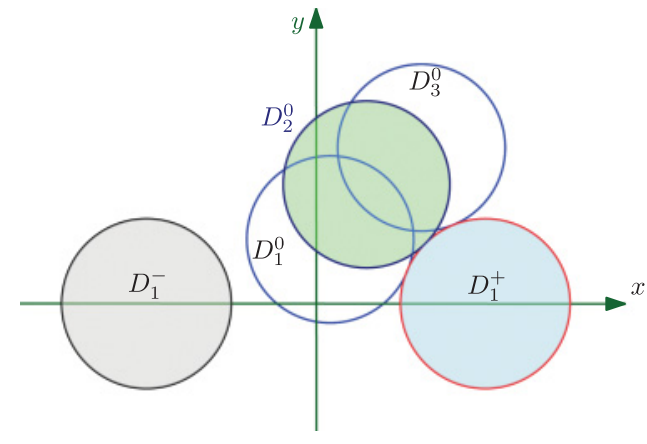

(a)

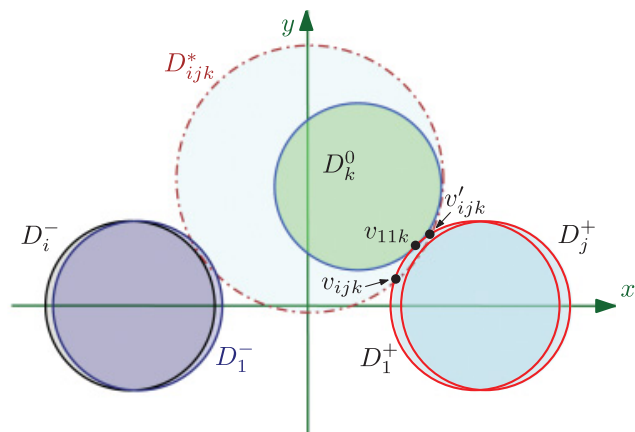

(b)

Fig. 5. (a) $\Omega\left(n^{3}\right)$ lower bound construction using disks of the same radius with $m=3$; only some disks are drawn. (b) Illustration of the proof.

Proof. Assume that $n=3 m$ for some $m \in \mathbb{N}^{+}$. We choose two parameters $\theta=\frac{\pi}{2} \cdot \frac{1}{(m+1)}$, and a sufficiently small positive number $\omega$. We construct three families of disks: $\mathcal{D}^{-}=$ $\left\{D_{1}^{-}, \ldots, D_{m}^{-}\right\}, \mathcal{D}^{+}=\left\{D_{1}^{+}, \ldots, D_{m}^{+}\right\}$, and $\mathcal{D}^{0}=\left\{D_{1}^{0}, \ldots, D_{m}^{0}\right\}$. Without loss of generality, we set the radius of all disks to 1 . The centers of disks in $\mathcal{D}^{-} \cup \mathcal{D}^{+}$lie on the $x$-axis, and the centers of disks in $\mathcal{D}^{0}$ lie in the first quadrant. More precisely, for $1 \leq i, j \leq m$, the center of $D_{i}^{-}$is $c_{i}^{-}=(-2-(i-1) \omega, 0)$ and the center of $D_{j}^{+}$is $c_{j}^{+}=(2+(j-1) \omega, 0)$, and for $1 \leq k \leq m$, the center of $D_{k}^{0}$ is $(2-2 \cos (k \theta), 2 \sin (k \theta))$. See Figure $5(\mathrm{a})$.

We claim that for every triple $i, j, k$ with $1 \leq i, j, k \leq m$, there is a disk touching $D_{i}^{-}$and $D_{j}^{+}$from the outside and $D_{k}^{0}$ from the inside and not containing any disk of $\mathcal{D}^{-} \cup \mathcal{D}^{+} \cup \mathcal{D}^{0}$ in its interior.

First of all, this is true for $i=j=1$ and $1 \leq k \leq m$. Note that $D_{1}^{-}$is centered at $(-2,0), D_{1}^{+}$is centered at $(2,0)$, and $D_{k}^{0}$ touches $D_{1}^{+}$from the outside. Since the radius of $D_{1}^{-}$and $D_{1}^{+}$is the same, the locus $b_{11}$ of the centers of disks that simultaneously touch $D_{1}^{-}$and $D_{1}^{+}$from the outside is the bisector of their centers; that is, $b_{11}$ is the $y$-axis. Fix a value $k$. It is easy to see that the disk $D_{11 k}^{*}$ centered at $(0,2 \tan (k \theta))$ with the radius $\frac{2}{\cos (k \theta)}-1$ touches $D_{1}^{-}$and $D_{1}^{+}$from the outside and $D_{k}^{0}$ from the inside. Furthermore, we show that $D_{11 k}^{*}$ does not contain disks in $\mathcal{D}^{0}$ in its interior (obvious for $\mathcal{D}^{-} \cup \mathcal{D}^{+}$). Since every disk in $\mathcal{D}^{0}$ touches $D_{1}^{+}$from the outside, $D_{11 k}^{*}$ containing a disk of $\mathcal{D}^{0}$ in its interior would imply that $D_{11 k}^{*}$ intersects the interior of $D_{1}^{+}$, a contradiction.

Next, we show that it holds for $1<i, j \leq m$ and $1 \leq k \leq m$. The key idea is that $D_{i}^{-}$( $D_{j}^{+}$, respectively) got placed by translating ("perturbing") $D_{1}^{-}$( $D_{1}^{+}$, respectively) so little that the disk $D_{i j k}^{*}$ touching $D_{i}^{-}$and $D_{j}^{+}$from the outside and $D_{k}^{0}$ from the inside does not contain any disk of $\mathcal{D}^{-} \cup \mathcal{D}^{+} \cup \mathcal{D}^{0}$ in its interior as for the case when $i=j=1$. We argue this using some elementary geometry. See Figure 5(b). Let $v_{i j k}$ and $v_{i j k}^{\prime}$ be the two intersection points of $\partial D_{i j k}^{*}$ and $\partial D_{1}^{+}$, for $1 \leq i, j, k \leq m$. Such two intersection points coincide with each other when $i=j=1$, and furthermore, they always exist due to our construction. Note that $v_{11 k}$ is also the intersection point of $\partial D_{k}^{0}$ and $\partial D_{1}^{+}$. It is trivial to see that as the parameter $\omega$ gets smaller, $v_{i j k}$ and $v_{i j k}^{\prime}$ lie closer to $v_{11 k}$ along $\partial D_{1}^{+}$. They all coincide with $v_{11 k}$ when $\omega$ becomes 0 . Since $\omega$ is a sufficiently small positive number, we are assured that $v_{i j k}$ lies between $v_{11(k-1)}$ and $v_{11 k}$ along $\partial D_{1}^{+}$; that is, $D_{i j k}^{*}$ does not contain $D_{k-1}^{0}$, not to mention $D_{1}^{0}, \ldots, D_{k-2}^{0}$. Similarly, $D_{i j k}^{*}$ does 
not contain $D_{k+1}^{0}, \ldots, D_{m}^{0}$. Moreover, $D_{i j k}^{*}$ does not contain any disk of $\mathcal{D}^{-} \cup \mathcal{D}^{+}$. Hence, there is a disk touching $D_{i}^{-}$and $D_{j}^{+}$from the outside and $D_{k}^{0}$ from the inside and not containing any disk of $\mathcal{D}^{-} \cup \mathcal{D}^{+} \cup \mathcal{D}^{0}$ in its interior, for $1 \leq i, j, k \leq m$.

This proves our claim and finishes our $\Omega\left(n^{3}\right)$ lower bound construction when the disks have the same radius.

We prove a refined bound on the complexity of $\mathcal{V}_{\neq 0}(\mathcal{P})$ if the uncertainty regions in $\mathcal{D}$ are pairwise-disjoint disks and the ratio of the radii of the largest to the smallest disk is bounded by $\lambda$.

LEMMA 2.8. If $\mathcal{P}=\left\{P_{1}, \ldots, P_{n}\right\}$ is a set of $n$ uncertain points in $\mathbb{R}^{2}$ whose uncertainty regions are pairwise-disjoint disks with radii in the range $[1, \lambda]$, a pair of curves in $\Gamma$ intersects in $O(\lambda)$ points.

PRoOF. Fix a pair of curves $\gamma_{1}$ and $\gamma_{2}$ in $\Gamma$. Let $D_{1}$ and $D_{2}$ be the disks corresponding to $\gamma_{1}$ and $\gamma_{2}$, and let $c_{1}$ and $c_{2}$ be their centers, respectively. By applying rotation and translation to the plane, we can assume $D_{1}$ and $D_{2}$ are centered on the $x$-axis, with $D_{1}$ to the left of $D_{2}$.

For a parameter $t, 1 \leq t \leq \lambda$, let $\mathcal{D}$ denote the set of all the disks associated with $\mathcal{P}$, excluding $D_{1}$ and $D_{2}$, with radii between $t$ and $2 t$. An intersection point $q \in \gamma_{1} \cap \gamma_{2}$ corresponds to a witness disk $W$ centered at $q$ that touches both $D_{1}$ and $D_{2}$ from the outside, touches exactly one other disk $E \in \mathcal{D}$ from the inside, and properly contains no disks of $\mathcal{D}$. The family of disks that touch both $D_{1}$ and $D_{2}$ from the outside is a pencil, which sweeps over a portion of the plane as the tangency points with $D_{1}$ and $D_{2}$ move continuously and monotonically in the $y$-direction. A disk of $\mathcal{D}$ can contribute at most two intersection points to $\gamma_{1} \cap \gamma_{2}$, as its boundary gets swept over at most twice by the circles of the pencil.

We break $\partial W$ into two curves, top and bottom, at W's tangency points with $D_{1}$ and $D_{2}$. For a disk $E \in \mathcal{D}$, if its tangency point with its witness disk $W$ is on the top portion of $W$, then it is a top tangency event; otherwise, it is a bottom tangency event. See Figure 6(a). Let $\mathcal{D}_{1}\left(\mathcal{D}_{2}\right.$, respectively) be the set of disks in $\mathcal{D}$ that are closer to $D_{1}\left(D_{2}\right.$, respectively).

Next we show that the number of top tangency events involving disks in $\mathcal{D}_{2}$ is $O(\lambda / t)$. Other tangency events are handled by a symmetric argument.

We remove from $\mathcal{D}_{2}$ all the disks within distance $T=\xi t$ from $D_{2}$, where $\xi$ is a sufficiently large constant. The ring with outer radius $\mathrm{r}\left(D_{2}\right)+4 T$ and inner radius $\mathrm{r}\left(D_{2}\right)$ has area

$$
\alpha=\pi\left(\left(\mathrm{r}\left(D_{2}\right)+4 T\right)^{2}-\left(\mathrm{r}\left(D_{2}\right)\right)^{2}\right)=O\left(T \mathrm{r}\left(D_{2}\right)+T^{2}\right)=O\left(t^{2}+\lambda t\right),
$$

as $r\left(D_{2}\right) \leq \lambda$. Disks removed from $\mathcal{D}_{2}$ have the following properties:

(i) They are interior disjoint.

(ii) Their radii lie in the interval $[t, 2 t]$.

(iii) They are contained in the aforementioned ring.

(iv) The area of each such disk is at least $\pi t^{2}$.

Hence, the number of removed disks is $O\left(\left(t^{2}+\lambda t\right) / t^{2}\right)=O(\lambda / t)$.

Consider the circle $\sigma_{2}$ of radius $\mathrm{r}\left(D_{2}\right)+T / 2$ centered at $c_{2}$. Consider any disk $E \in \mathcal{D}_{2}$ and its witness disk $W$ touching both $D_{1}$ and $D_{2}$ from the outside. If $E$ has not been removed from $\mathcal{D}_{2}$, then $r(W) \geq(T+2 t) / 2$; in particular, it is larger than $T / 2$ and the center of $W$ lies outside $\sigma_{2}$. Let $W_{\ominus \tau}$ be the disk concentric with $W$ with radius $\mathrm{r}(W)-\tau$, where $\tau=4 t$. The interior of $W_{\ominus \tau}$ is disjoint from all disks in $\mathcal{D}_{2}$, as $E$ touches $W$ from inside and $W$ does not fully contain any other disks from $\mathcal{D}_{2}$. 


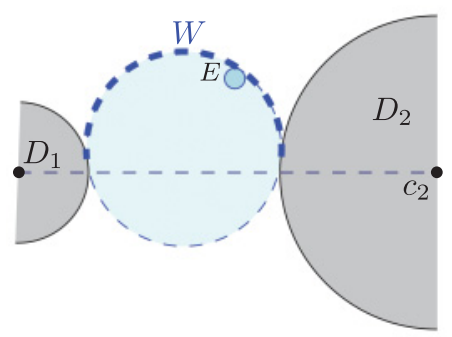

(a)

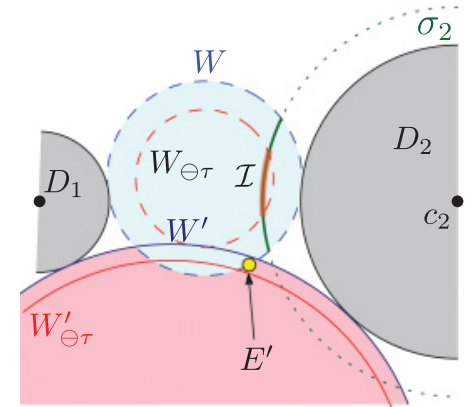

(c)

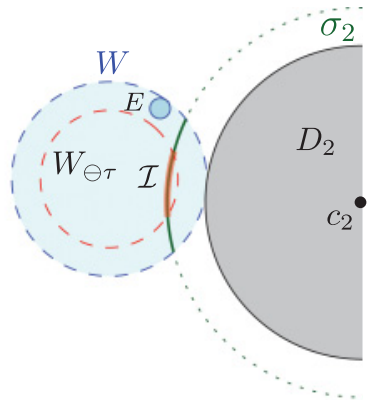

(b)

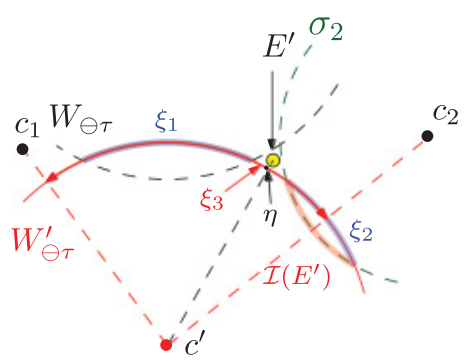

(d)

Fig. 6. An illustration for the proof of Lemma 2.8.

The witness disk $W$ covers an arc of length at least $T / 2$ on $\sigma_{2}$. Indeed, neither of these two disks contains the center of the other, and the inner distance between the two intersection arcs is $T / 2$ (see figure on the right). Similarly, let $\mathcal{J}(E)$ be the arc $W_{\ominus \tau} \cap \sigma_{2}$. By the same argument, we have that $\mathcal{J}(E)$ is of length at least $T / 2-\tau=\Omega(t)$.

The circumference of $\sigma_{2}$ is $2 \pi\left(\mathrm{r}\left(D_{2}\right)+T / 2\right)=O(\lambda)$, so if the $\operatorname{arcs} \mathcal{J}(E)$, for $E \in \mathcal{D}_{2}$, are pairwise disjoint, we are done, as this implies that there could be at most $\lambda /(T / 2-\tau)=O(\lambda / t)$ such arcs and thus the size of the original $\mathcal{D}_{2}$, including the disks that were deleted from $\mathcal{D}_{2}$, is $O(\lambda / t)$. See Figure 6(b).

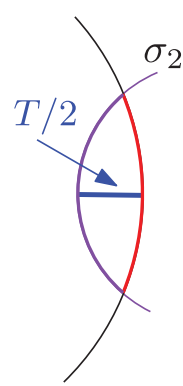

We now prove the claim that for any two disks $E, E^{\prime} \in \mathcal{D}_{2}$ realizing a top tangency event, $\mathcal{J}(E)$ and $\mathcal{J}\left(E^{\prime}\right)$ are disjoint.

Let $W$ ( $W^{\prime}$, respectively) be the witness disk that is tangent to $D_{1}, D_{2}$, and $E$ ( $E^{\prime}$, respectively). Assume that the tangency of $W$ with $D_{2}$ is clockwise to the tangency of $W^{\prime}$ with $D_{2}$ (i.e., $E$ is "above" $E^{\prime}$ ). If $W_{\ominus \tau}$ and $W_{\ominus \tau}^{\prime}$ are disjoint, then the corresponding $\operatorname{arcs} \mathcal{J}(E)$ and $\mathcal{J}\left(E^{\prime}\right)$ are obviously disjoint, so assume that $W_{\ominus \tau}$ and $W_{\ominus \tau}^{\prime}$ intersect; see Figure 6(c).

Let $c^{\prime}$ be the center of $W_{\ominus \tau}^{\prime}$. We define three circular arcs on $\partial W_{\ominus \tau}^{\prime}$. Let $\xi_{1}=\partial W_{\ominus \tau}^{\prime} \cap$ $W_{\ominus \tau}$, let $\xi_{2}$ be the portion of $\partial W_{\ominus \tau}^{\prime}$ lying in the disk bounded by $\sigma_{2}$, and let $\xi_{3}$ be the portion of $\partial W_{\ominus \tau}^{\prime}$ lying in the wedge formed by the rays $c^{\prime} c_{1}$ and $c^{\prime} c_{2}$; see Figure $6(\mathrm{~d})$. It can be verified that $\xi_{1} \subset \xi_{3}$ and the right endpoint of $\xi_{3}$ lies inside $\sigma_{2}$ and thus on $\xi_{2}$.

Next, let $\eta \in \partial W_{\ominus \tau}^{\prime}$ be the intersection point of $\partial W_{\ominus \tau}^{\prime}$ with the segment connecting $c^{\prime}$ and the center of $E^{\prime}$; since $E^{\prime}$ lies in the exterior of $W_{\ominus \tau}^{\prime}, \eta$ exists. Since $E^{\prime}$ realizes a top tangency event, $\eta \in \xi_{3}$. Furthermore, $E^{\prime}$ lies in the exterior of $W_{\ominus \tau}$ and $E^{\prime} \in \mathcal{D}_{2}$, which implies that $\eta \notin \xi_{1}$ and it lies to the right of $\xi_{1}$. Similarly, $E^{\prime}$ lies in the exterior of $\sigma_{2}$ 


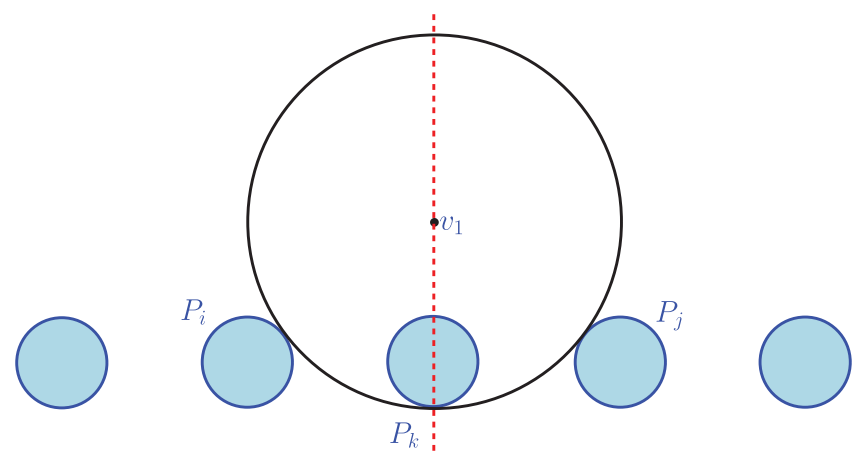

Fig. 7. Any pair $\left(P_{i}, P_{j}\right)$ satisfying $j-i \geq 2$ determines two vertices of $\mathcal{V}_{\neq 0}$. Only the vertex $v_{1}$ is shown.

and the right endpoint of $\xi_{3}$ lies on $\xi_{2}$; therefore, $\eta$ lies to the left of the arc $\xi_{2}$. In other words, $\eta$ separates $\xi_{1}$ and $\xi_{2}$, implying that $\xi_{1} \cap \xi_{2}=\varnothing$, which in turn implies that the top endpoint of $\mathcal{J}\left(E^{\prime}\right)$ does not lie inside $W_{\ominus \tau}$. Hence, $\mathcal{J}(E) \cap \mathcal{J}\left(E^{\prime}\right)=\emptyset$, as claimed.

Finally, we repeat the aforementioned counting argument, for $t=1,2,4, \ldots, 2^{m}$, where $m=\left\lceil\log _{2} \lambda\right\rceil$, concluding that the number of intersection points between $\gamma_{1}$ and $\gamma_{2}$ is bounded by $\sum_{i=1}^{m} O\left(\lambda / 2^{i}\right)=O(\lambda)$. This completes the proof of the lemma.

TheOREM 2.9. Let $\mathcal{P}=\left\{P_{1}, \ldots, P_{n}\right\}$ be a set of $n$ uncertain points in $\mathbb{R}^{2}$ such that their uncertainty regions are pairwise-disjoint disks and that the ratio of the largest and the smallest radii of the disks is at most $\lambda$. Then, the complexity of $\mathcal{V}_{\neq 0}(\mathcal{P})$ is $O\left(\lambda n^{2}\right)$, and it can be computed in $O\left(n^{2} \log n+\mu\right)$ expected time, where $\mu$ is the complexity of $\mathcal{V}_{\neq 0}(\mathcal{P})$. Furthermore, there exists such a set $\mathcal{P}$ of uncertain points for which $\nu_{\neq 0}(\mathcal{P})$ has $\Omega\left(n^{2}\right)$ complexity.

Proof. The upper bound on the complexity of $\mathcal{V}_{\neq 0}(\mathcal{P})$ follows from Lemma 2.8. By the same argument as in the proof of Theorem 2.5, $\mathcal{V}_{\neq 0}(\mathcal{P})$ can be computed in $O\left(n^{2} \log n+\mu\right)$ time, where $\mu$ is the number of vertices in $\mathcal{V}_{\neq 0}(\mathcal{P})$.

Next we show that there exists a set $\mathcal{P}$ of $n$ uncertain points in $\mathbb{R}^{2}$ such that $\mathcal{V}_{\neq 0}(\mathcal{P})$ has $\Omega\left(n^{2}\right)$ vertices. Assume that $n=2 m$ for some positive integer $m$. All the disks $D_{i}$ have the same radius 1 , centered at $c_{i}=(4(i-m)-2,0)$, for $1 \leq i \leq 2 m$. Any pair $\left(P_{i}, P_{j}\right)$ satisfying that $j-i \geq 2$ and $j+i$ is even determines two vertices: $v_{1}=\left(2(i+j-2 m-1),(j-i)^{2}-1\right)$, and $v_{2}=\left(2(i+j-2 m-1), 1-(j-i)^{2}\right)$, of $\mathcal{V}_{\neq 0}$ (realized with $\left.P_{k}, k=\frac{j+i}{2}\right)$ (Figure 7). Any pair $\left(P_{i}, P_{j}\right)$ satisfying that $j-i \geq 2$ and $j+i$ is odd determines two vertices: $v_{1}=\left(2(i+j-2 m-1),(j-i) \sqrt{(j-i)^{2}-4}\right)$, and $v_{2}=\left(2(i+j-2 m-1),(i-j) \sqrt{(j-i)^{2}-4}\right)$, of $\mathcal{V}_{\neq 0}$ (realized with $P_{k}, k=\left\lfloor\frac{j+i}{2}\right\rfloor$ or $k=\left\lceil\frac{j+i}{2}\right\rceil$ ). One can verify that $\delta_{i}(v)=\delta_{j}(v)=$ $\Delta_{k}(v) \leq \Delta_{l}(v)$, for $1 \leq l \leq n, v \in\left\{v_{1}, v_{2}\right\}$. Hence, we obtain a lower bound of $\Omega\left(n^{2}\right)$ for the complexity of $\mathcal{V}_{\neq 0}$.

Remarks. We note that the proof of Lemma 2.8 is essentially a packing argument, and therefore can be extended to the case when each uncertainty region is a convex $\alpha$-fat semialgebraic set of constant description complexity. A convex set $C$ is called $\alpha$-fat if there exist two concentric disks $D$ and $D^{\prime}$ so that $D \subseteq C \subseteq D^{\prime}$ and the ratio between the radii of $D^{\prime}$ and $D$ is at most $\alpha$. The constant of proportionality also depends on $\alpha$ and the description complexity of the sets defining the uncertainty regions. This in turn implies that $\nu_{\neq 0}(\mathcal{P})$ has $O\left(\lambda n^{2}\right)$ complexity if the uncertainty regions of $\mathcal{P}$ are pairwise-disjoint convex $\alpha$-fat sets, for some constant $\alpha \geq 1$, and the ratio of the size of the largest to the smallest region is bounded by $\lambda$. Extension of the proof of Lemma 2.8 to this case, 
however, is even more technical, so we have decided not to state this generalized result as a theorem, especially since, in practice, a fat convex set can be approximated by a circular disk.

Storing $\mathcal{P}_{\phi} s$ for $\mathcal{V}_{\neq 0}(\mathcal{P})$. We store the index $i$ of each uncertain point $P_{i}$ instead of $P_{i}$ itself. If we store $\mathcal{P}_{\phi}$ for each cell $\phi$ of $\mathcal{V}_{\neq 0}(\mathcal{P})$ explicitly, the size increases by a factor of $n$. However, we observe that for two adjacent cells $\phi, \phi^{\prime}$ of $\mathcal{V}_{\neq 0}(\mathcal{P})$, that is, two cells that share a common edge, $\left|\mathcal{P}_{\phi} \oplus \mathcal{P}_{\phi^{\prime}}\right|=1$, where $\oplus$ denotes the symmetric difference of two sets. Therefore, using a persistent data structure [Driscoll et al. 1989], we can store $\mathcal{P}_{\phi}$ for all cells of $\mathcal{V}_{\neq 0}(\mathcal{P})$ in $O(\mu)$ space, where $\mu$ is the complexity of $\mathcal{V}_{\neq 0}(\mathcal{P})$, so that for any cell $\phi, \mathcal{P}_{\phi}$ can be retrieved in $O\left(\log n+\left|\mathcal{P}_{\phi}\right|\right)$ time. ${ }^{3}$ By combining this with a planar point-location data structure [de Berg et al. 2008], we obtain the following:

TheOREm 2.10. Let $\mathcal{P}$ be a set of $n$ uncertain points in $\mathbb{R}^{2}$, and let $\mu$ be the complexity of $\mathcal{V}_{\neq 0}(\mathcal{P})$. Then, $\mathcal{V}_{\neq 0}(\mathcal{P})$ can be preprocessed in $O(\mu \log \mu)$ time into a data structure of size $O(\mu)$ so that, for a query point $q \in \mathbb{R}^{2}, N N_{\neq 0}(q, \mathcal{P})$ can be computed in $O(\log n+t)$ time, where $t$ is the output size.

\subsection{Discrete Case}

We now analyze the complexity of $\mathcal{V}_{\neq 0}(\mathcal{P})$ when the distribution of each point $P_{i}$ in $\mathcal{P}$ is discrete. Let $P_{i}=\left\{p_{i 1}, \ldots, p_{i k}\right\}$. For $1 \leq j \leq k$, let $w_{i j}=\operatorname{Pr}\left[P_{i}\right.$ is $\left.p_{i j}\right]$. As in the previous section, for a point $x$, let

$$
\Delta_{i}(q)=\max _{1 \leq j \leq k} d\left(q, p_{i j}\right) \quad \text { and } \quad \delta_{i}(q)=\min _{1 \leq j \leq k} d\left(q, p_{i j}\right) .
$$

Note that the projection of the graph of $\Delta_{i}\left(\delta_{i}\right.$, respectively) onto the $x y$-plane is the farthest-point (nearest-point, respectively) Voronoi diagram of $P_{i}$. Let $\Delta(q)=$ $\min _{1 \leq i \leq n} \Delta_{i}(q)$. For each $i$, let $\gamma_{i}=\left\{x \in \mathbb{R}^{2} \mid \delta_{i}(x)=\Delta(x)\right\}$, and set $\Gamma=\left\{\gamma_{1}, \ldots, \gamma_{n}\right\}$. Then $\mathcal{V}_{\neq 0}(\mathcal{P})$ is the planar subdivision $\mathcal{A}(\Gamma)$ induced by $\Gamma$ (cf. Corollary 2.4).

We define a few functions that will help analyze the structure of $\mathcal{V}_{\neq 0}(\mathcal{P})$. We first define a function $f: \mathbb{R}^{2} \times \mathbb{R}^{2} \rightarrow \mathbb{R}$ as

$$
f(x, p)=d^{2}(x, p)-\|x\|^{2}=\|p\|^{2}-2\langle x, p\rangle .
$$

For $1 \leq i \leq n$, define

$$
\varphi_{i}(x)=\min _{1 \leq j \leq k} f\left(x, p_{i j}\right) \quad \text { and } \quad \Phi_{i}(x)=\max _{1 \leq j \leq k} f\left(x, p_{i j}\right) .
$$

Finally, we define

$$
\Phi(x)=\min _{1 \leq i \leq n} \Phi_{i}(x) .
$$

The following lemma is straightforward.

Lemma 2.11. For any $i \leq n$ and for any $q \in \mathbb{R}^{2}, \delta_{i}(q)=r$ if and only if $\varphi_{i}(q)=r^{2}-\|q\|^{2}$.

Lemma 2.12. For any pair $i, j, 1 \leq i \neq j \leq n$, let $\gamma_{i j}=\left\{x \in \mathbb{R}^{2} \mid \delta_{i}(x)=\Delta_{j}(x)\right\}$, and then $\gamma_{i j}$ is a convex polygonal curve with $O(k)$ vertices.

Proof. By Lemma 2.11, for any pair $i, j$ and for any $x \in \mathbb{R}^{2}, \delta_{i}(x)=\Delta_{j}(x)$ if and only if $\varphi_{i}(x)=\Phi_{j}(x)$. Hence, $\gamma_{i j}$ is also the zero set of the function $\Phi_{j}(x)-\varphi_{i}(x)$.

\footnotetext{
${ }^{3}$ If the curves of $\Gamma$ intersect transversally at every vertex, it suffices to store $\mathcal{P}_{\phi}$ for each cell of $\mathcal{V}_{\neq 0}(\mathcal{P})$. Otherwise, one may have to store $\mathcal{P}_{\phi}$ for edges and vertices of $\mathcal{V}_{\neq 0}(\mathcal{P})$. This does not affect the asymptotic performance of the data structure.
} 
$\Phi_{j}$ is the upper envelope of $k$ linear functions, and thus is a piecewise-linear convex function. Similarly, $\varphi_{i}$, the lower envelope of $k$ linear functions, is a piecewise-linear concave function. Hence, $\Phi_{j}(x)-\varphi_{i}(x)$ is a piecewise-linear convex function, which implies that $\gamma_{i j}=\left\{x \in \mathbb{R}^{2} \mid \Phi_{j}(x)=\varphi_{i}(x)\right\}$ is a convex polygonal curve. Since $\gamma_{i j}$ is the projection of the intersection curve of the graphs of $\Phi_{j}$ and $\varphi_{i}$, each of which is the surface of an unbounded convex polyhedron with at most $k$ faces, $\gamma_{i j}$ has $O(k)$ vertices.

Theorem 2.13. Let $\mathcal{P}=\left\{P_{1}, \ldots, P_{n}\right\}$ be a set of $n$ uncertain points in $\mathbb{R}^{2}$, where each $P_{i}$ has a discrete distribution of size at most $k$. The complexity of $\mathcal{V}_{\neq 0}(\mathcal{P})$ is $\mu=O\left(k n^{3}\right)$, and it can be computed in $O\left(n^{2} \log n+\mu\right)$ expected time. Furthermore, it can be preprocessed in additional $O(\mu)$ time into a data structure of size $O(\mu)$ so that an $N N_{\neq 0}(q)$ query can be answered in $O(\log \mu+t)$, where $t$ is the output size.

Proof. We follow the same argument as in the proof of Theorem 2.5. We need to bound the number of intersection points between a pair of curves $\gamma_{i}$ and $\gamma_{j}$. Fix an index $u$. Let $\gamma_{i u}=\left\{x \in \mathbb{R}^{2} \mid \delta_{i}(x)=\Delta_{u}(x)\right\}$ and $\gamma_{j u}=\left\{x \in \mathbb{R}^{2} \mid \delta_{j}(x)=\Delta_{u}(x)\right\}$. By Lemma 2.12, each of $\gamma_{i u}$ and $\gamma_{j u}$ is a convex polygonal curve in $\mathbb{R}^{2}$ with $O(k)$ vertices. Since two convex polygonal curves in general position with $n_{1}$ and $n_{2}$ vertices intersect in at most $n_{1}+n_{2}$ points, $\gamma_{i u}$ and $\gamma_{j u}$ intersect at $O(k)$ points. Hence, $\gamma_{i}$ and $\gamma_{j}$ intersect at $O(k n)$ points, implying that $\mathcal{V}_{\neq 0}(\mathcal{P})$ has $O\left(k n^{3}\right)$ vertices. The running time follows from the proof of Theorem 2.5.

\section{DATA STRUCTURES FOR $\mathrm{NN}_{\neq 0}$ QUERIES}

With the maximum size of $\mathcal{V}_{\neq 0}$ being $\Theta\left(n^{3}\right)$, we present $O(n$ polylog $(n))$-size data structures that circumvent the need for constructing $\nu_{\neq 0}(\mathcal{P})$ and answer $N N_{\neq 0}$ queries in polylogarithmic or sublinear time. They rely on geometric data structures for answering range-searching queries and their variants; see Agarwal [2016] for a recent survey.

An $\mathrm{NN}_{\neq 0}(q)$ query is answered in two stages. The first stage computes $\Delta(q)$, and the second stage computes all points $P_{i} \in \mathcal{P}$ for which $\delta_{i}(q)<\Delta(q)$. We build a separate data structure for each stage. We first describe the one for the continuous case and then for the discrete case.

Continuous Case. We assume that the uncertainty region of each point $P_{i}$ is a disk $D_{i}$ of radius $r_{i}$ centered at $c_{i}$. Recall from Section 2 that the projection of the graph of the function $\Delta$ onto the $x y$-plane, a planar subdivision $\mathbb{M}$, is the (additive-weighted) Voronoi diagram of the points $c_{1}, \ldots, c_{n}$, and it has linear complexity. Hence, $\mathbb{M}$ can be preprocessed in $O(n \log n)$ time into a data structure of size $O(n)$ so that for a query point $q \in \mathbb{R}^{2}, \Delta(q)$ can be computed in $O(\log n)$ time [de Berg et al. 2008].

Next we wish to report all points $P_{i} \in \mathcal{P}$ for which $\delta_{i}(q)<\Delta(q)$, that is, for which $D_{i}$ intersects the disk of radius $\Delta(q)$ centered at $q$. Note that the projection of the graph of the lower envelope of $\left\{\delta_{1}, \ldots, \delta_{n}\right\}$ is also an (additive-weighted) Voronoi diagram of the points $c_{1}, \ldots, c_{n}$ and has linear complexity. Recently Kaplan et al. [2016] have described a data structure of size $O(n$ polylog $(n))$ that can answer the previous query in $O(\log n+t)$ time, where $t$ is the output size. It can be constructed in $O(n$ polylog $(n))$ randomized expected time. We thus obtain the following:

Theorem 3.1. Let $\mathcal{P}=\left\{P_{1}, \ldots, P_{n}\right\}$ be a set of $n$ uncertain points in $\mathbb{R}^{2}$ so that the uncertainty region of each $P_{i}$ is a disk. $\mathcal{P}$ can be preprocessed into a data structure of size $O(n$ polylog $(n))$, so that an $N N_{\neq 0}(q)$ query can be answered in $O(\log n+t)$ time, where $t$ is the output size. The data structure can be constructed in $O(n$ polylog $(n))$ randomized expected time. 
Remarks. (i) Note that Theorem 3.1 gives a better result than Theorem 2.10 but the data structure based on $\mathcal{V}_{\neq 0}(\mathcal{P})$ is simpler and more practical.

(ii) If we use the $L_{1}$ or $L_{\infty}$ metric to compute the distance between points and use disks in the $L_{1}$ or $L_{\infty}$ metric (i.e., a diamond or a square), then an $\mathrm{NN}_{\neq 0}(q)$ query can be answered in $O\left(\log ^{2} n+t\right)$ time using $O\left(n \log ^{2} n\right)$ space: the first stage remains the same and the second stage reduces to reporting a set of axis-aligned squares that intersect a query axis-aligned square [Agarwal 2016].

Discrete Case. Next, we consider the case when each $P_{i}$ has a discrete distribution of size at most $k$; set $N=n k$. The functions $\Delta_{i}$ and $\delta_{i}$ are now more complex and thus the data structure for $\mathrm{NN}_{\neq 0}(q)$ queries is more involved. As in Section 2.2, instead of working with the functions $\delta_{i}$ and $\Delta_{i}$, we work with $\varphi_{i}$ and $\Phi_{i}$. By Lemma 2.11, the problem of reporting all points $P_{i}$ with $\delta_{i}(q)<\Delta(q)$ is equivalent to returning the points with $\varphi_{i}(q)<\Phi(q)$. As for the continuous case, we construct two data structures-the first one computes $\Phi(q)$ for a query point $q \in \mathbb{R}^{2}$ and the second one reports all $P_{i}$ s with $\varphi_{i}(q)<\Phi(q)$. Note that $\varphi_{i}(q)<\Phi(q)$ if and only if the point $\widehat{q}=(q, \Phi(q)) \in \mathbb{R}^{3}$ lies above the graph of $\varphi_{i}$. By triangulating each face of $\varphi_{i}$ and $\Phi_{i}$ if necessary, we can assume that each $\varphi_{i}$ is a triangulated concave surface and each $\Phi_{i}$ is a triangulated convex surface.

We now describe the data structure for computing $\Phi(q)$. Note that $\Phi(q)=\Phi_{j}(q)$ if $\Phi_{j}$ is the first surface in the set $\left\{\Phi_{1}, \ldots, \Phi_{n}\right\}$ intersected by $\ell_{q}$, the vertical line passing through $q$, in the $(+z)$-direction. We first construct a three-level partition tree [Agarwal $2016]$ on the set of triangles in the graphs of $\Phi_{1}, \ldots, \Phi_{n}$, denoted by $\Sigma$, so that the triangles of $\Sigma$ intersected by $\ell_{q}$, for a query point $q \in \mathbb{R}^{2}$, can be reported efficiently. The partition tree stores a family of canonical subsets of triangles in $\Sigma$ so that for any query point $q$, the triangles of $\Sigma$ intersected by $\ell_{q}$ can be reported as the union of $O\left(\sqrt{N} \log ^{2} n\right)$ canonical subsets in $O\left(\sqrt{N} \log ^{2} n\right)$ time. Let $F_{q}$ denote the family of canonical subsets reported by the query procedure. The size of the data structure is $O\left(N \log ^{2} n\right)$, and it can be constructed in $O\left(N \log ^{2} N\right)$ randomized expected time [Agarwal 2016]. Next, for each canonical subset $C$, let $C^{*}$ be the set of planes supporting the triangles in $C$. We construct the lower envelope $L_{C}$ of $C^{*}$ (by regarding each plane in $C^{*}$ as the graph of a linear function), which has size $O(|C|)$, and preprocess $L_{C}$ into an $O(|C|)$ size data structure so that for a query point $q \in \mathbb{R}^{2}, L_{C}(q)$ can be computed in $O(\log |C|)$ time [Sharir and Agarwal 1995]. Summing over all canonical subsets of the partition tree, the overall size of the data structure is $O\left(N \log ^{2} N\right)$ and it can be constructed in $O\left(N \log ^{3} N\right)$ randomized expected time.

Given a query point $q \in \mathbb{R}^{2}$, we first query the partition tree and compute the family $F_{q}$ of canonical subsets. For each canonical set $C \in F_{q}$, we compute $L_{C}(q)$ and return the minimum among them as $\Phi(q)$. Since the procedure spends $O(\log N)$ time for each canonical subset, the overall query time is $O\left(\sqrt{N} \log ^{3} N\right)$. The correctness of the procedure follows from the following observation: $\ell_{q}$ intersects all triangles of a canonical subset $C \in F_{q}$, so for each triangle $\tau \in C$ and its supporting plane $\tau^{*}$, $\ell_{q} \cap \tau=\ell_{q} \cap \tau^{*}$. Therefore, $L_{C}(q)$ is the same as the (height of the) first intersection point of $\ell_{q}$ with a triangle of $C$, and $\Phi(q)=\min _{C \in F_{q}} L_{C}(q)$.

Next, we describe the data structure for reporting the points $P_{i}$ with $\varphi_{i}(q)<\Phi(q)$. It is very similar to the one just described, except for one twist. First, as earlier, we construct a three-level partition tree on the triangles in the graphs of $\varphi_{1}, \ldots, \varphi_{n}$. Let $C$ be a canonical subset constructed by the partition tree, and let $C^{*}$ be the set of planes supporting $C$. Using a result by Afshani and Chan [2009] (see also Agarwal [2016]), we preprocess $C^{*}$, in $O(|C| \log |C|)$ randomized expected time, into a data structure of size $O\left(\left|C^{*}\right|\right)$ so that for a query point $\widehat{q}=(q, \Phi(q))$, all $t_{C}$ planes of $C^{*}$ lying below $\hat{q}$ can 
be reported in $O\left(\log N+t_{C}\right)$ time. Summing over all canonical subsets of the partition tree, the overall size of the data structure is $O\left(N \log ^{2} N\right)$, and it can be constructed in $O\left(N \log ^{3} N\right)$ randomized expected time.

Given a query point $q \in \mathbb{R}^{2}$, we first query the partition tree and compute the family $F_{q}$ of canonical subsets. For each canonical set $C \in F_{q}$, we next report all planes of $C^{*}$ lying below $\hat{q}$. The overall query time is $O\left(\sqrt{N} \log ^{3} N+t\right)$, where $t$ is the output size. ${ }^{4}$ The correctness of the procedure follows from the same argument as earlier-namely, since $\ell_{q}$ intersects all triangles of a canonical subset $C \in F_{q}$, a triangle in $C$ lies below $\widehat{q}$ if and only if the plane supporting it lies below $\widehat{q}$.

Putting everything together, we can construct, in $O\left(N \log ^{3} N\right)$ randomized expected time, a data structure of $O\left(N \log ^{2} N\right)$ size that can answer an $\mathrm{NN}_{\neq 0}$ query in $O\left(\sqrt{N} \log ^{3} N\right)$ time.

Finally, we remark that the three-level partition tree can be replaced by a multilevel data structure of size $O\left(N^{2} \log ^{2} N\right)$ so that the set of triangles intersected by $\ell_{q}$ can be returned as the union of $O\left(\log ^{3} N\right)$ canonical subsets [Agarwal 2016]. Using this data structure, we can answer an $\mathrm{NN}_{\neq 0}$ query in $O\left(\log ^{4} N\right)$ time using $O\left(N^{2} \log ^{2} N\right)$ space. We thus obtain the following:

THEOREM 3.2. Let $\mathcal{P}$ be a set of $n$ uncertain points in $\mathbb{R}^{2}$, each with a discrete distribution of size at most $k$; set $N=n k$. $\mathcal{P}$ can be preprocessed into a data structure of size $O\left(N \log ^{3} N\right)$ so that an $N N_{\neq 0}(q)$ query can be answered in $O\left(\sqrt{N} \log ^{3} N+t\right)$ time, or into a data structure of size $O\left(N^{2} \log ^{2} N\right)$ with $O\left(\log ^{4} N+t\right)$ query time, where $t$ is the output size. The expected preprocessing times are $O\left(N \log ^{3} N\right)$ and $O\left(N^{2} \log ^{3} N\right)$ time, respectively.

\section{QUANTIFICATION PROBABILITIES}

We now turn our attention to the second part of answering probabilistic NN queries, namely, returning the quantification probabilities that are positive. We begin with a data structure for computing quantification probabilities exactly for the case when each uncertain point has a discrete distribution of size at most $k$. Since computing these quantities exactly is quite expensive and they are small for most of the points, we focus on computing quantification probabilities approximately.

\subsection{The Exact Algorithm}

Assuming each point in $\mathcal{P}$ has a discrete distribution of size at most $k$, we build the probabilistic Voronoi diagram $\mathcal{V}_{\operatorname{Pr}}(\mathcal{P})$ that decomposes $\mathbb{R}^{2}$ into a set of cells, so that any point $q$ in a cell has the same $\pi_{i}(q)$ value for all $P_{i} \in \mathcal{P}$; that is, for any point $q$ in this cell, we know exactly the probability of each point $P \in \mathcal{P}$ being the NN of $q$.

Lemma 4.1. Let $\mathcal{P}$ be a set of $n$ uncertain points in $\mathbb{R}^{2}$, each with a discrete distribution of size at most $k$; set $N=n k$. The complexity of $\mathcal{V}_{\operatorname{Pr}}(\mathcal{P})$ is $O\left(N^{4}\right)$. Moreover, there exists a set $\mathcal{P}$ of $n$ uncertain points in $\mathbb{R}^{2}$ with $k=2$ such that $\mathcal{V}_{\operatorname{Pr}}(\mathcal{P})$ has size $\Omega\left(n^{4}\right)$.

Proof. We first prove the upper bound. There are $N$ possible locations. Each pair of possible locations determines a bisector, resulting in $O\left(N^{2}\right)$ bisectors. These bisectors partition the plane into $O\left(N^{4}\right)$ convex cells so that the order of all distances to each of

\footnotetext{
${ }^{4}$ We note that if $\ell_{q}$ passes through the boundary of a triangle of some $\varphi_{i}$, then $P_{i}$ may be reported multiple times. If the points of $P_{i}$ are in general position, then the degree of each vertex of $\varphi_{i}$ is constant, so $P_{i}$ will be reported $O(1)$ times. However, if points in $P_{i}$ are in a degenerate position, then additional care is needed, using standard techniques such as symbolic perturbation, to ensure that $P_{i}$ is reported only $O(1)$ times.
} 


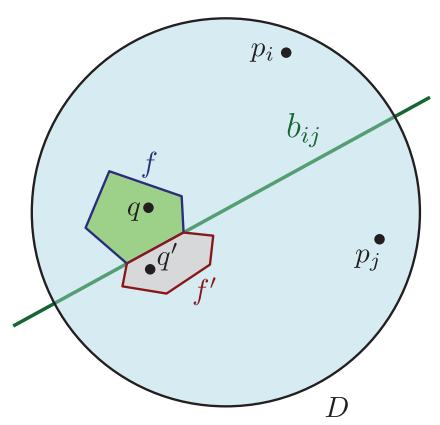

Fig. 8. An illustration of the proof. Inside the unit disk $D$, two adjacent faces $f$ and $f^{\prime}$ share a portion of the bisector $b_{i j}$ defined by $p_{i}$ and $p_{j}$.

the $n k$ possible locations, and thus by Equation (2) also all the quantification probabilities, are preserved within each cell. Therefore, the resulting planar subdivision is a refinement of $\mathcal{V}_{\operatorname{Pr}}(\mathcal{P})$, and thus $O\left(n^{4} k^{4}\right)$ is an upper bound on the complexity of $\mathcal{V}_{\operatorname{Pr}}(\mathcal{P})$.

Next, we show that there exists a set $\mathcal{P}$ of $n$ uncertain points in $\mathbb{R}^{2}$ with $k=2$ such that $\mathcal{V}_{\operatorname{Pr}}(\mathcal{P})$ has size $\Omega\left(n^{4}\right)$. For simplicity, we describe a degenerate configuration of points, but the argument can be generalized to a nondegenerate configuration as well, by being more careful. For every $1 \leq i \leq n, P_{i} \in \mathcal{P}$ has two possible locations $p_{i}$ and $p_{i}^{\prime}$, each with probability 0.5 . Let $D$ be the unit disk centered at the origin. We choose $p_{1}, \ldots, p_{n}$ inside $D$ so that the bisector $b_{i j}$ of every pair $p_{i}, p_{j}$, for $i<j$, is a distinct line and all pairs of bisectors intersect inside $D$. We place all $p_{i}^{\prime} \mathrm{s}$ at the same location far away from $D$, say, at $\bar{p}=(100,0)$. Note that the bisector of $\bar{p}$ and $p_{i}$, for any $i \leq n$, does not intersect $D$, so for any point $q \in D, d\left(p_{i}, q\right)<d(\bar{p}, q)$.

Let $\mathcal{A}$ be the arrangement of the bisectors $\left\{b_{i j} \mid 1 \leq i<j \leq n\right\}$. Since all pairs of bisectors intersect inside $D, \mathcal{A} \cap D$ has $\Theta\left(n^{4}\right)$ faces. Let $f, f^{\prime}$ be any two adjacent faces of $\mathcal{A}$ inside $D$, let $b_{i j}$ be the bisector separating $f$ and $f^{\prime}$, and let $q, q^{\prime}$ be arbitrary points in the interior of $f, f^{\prime}$, respectively. Without loss of generality, assume that $d\left(p_{i}, q\right)<d\left(p_{j}, q\right)$, and then $d\left(p_{i}, q^{\prime}\right)>d\left(p_{j}, q^{\prime}\right)$. See Figure 8. Suppose there are $r$, $0 \leq r<n-1$, points of $\left\{p_{1}, \ldots, p_{n}\right\}$ that are closer to $q$ than $p_{i}$; that is, $p_{i}\left(p_{j}\right.$, respectively) is the $(r+1)$-st NN of $q\left(q^{\prime}\right.$, respectively) among $\left\{p_{1}, \ldots, p_{n}\right\}$. Then, by Equation (2),

$$
\begin{aligned}
& \pi_{i}(q)=0.5 \cdot(1-0.5)^{r}+0.5 \cdot(1-0.5)^{n-1}=0.5^{r+1}+0.5^{n}, \text { and } \\
& \pi_{j}(q)=0.5 \cdot(1-0.5)^{r+1}+0.5 \cdot(1-0.5)^{n-1}=0.5^{r+2}+0.5^{n} .
\end{aligned}
$$

Symmetrically, $\pi_{i}\left(q^{\prime}\right)=0.5^{r+2}+0.5^{n}$ and $\pi_{j}\left(q^{\prime}\right)=0.5^{r+1}+0.5^{n}$. In particular, $\pi_{i}(q) \neq$ $\pi_{i}\left(q^{\prime}\right)$ and $\pi_{j}(q) \neq \pi_{j}\left(q^{\prime}\right)$. In other words, any two adjacent faces of $\mathcal{A}$ inside $D$ have distinct quantification probability vectors, implying that $\mathcal{V}_{\operatorname{Pr}}(\mathcal{P})$ has $\Omega\left(n^{4}\right)$ complexity.

As in Section 2.1, we can store the quantification probabilities for all faces of $\mathcal{V}_{\operatorname{Pr}}(\mathcal{P})$ by using $O(1)$ storage per face. Hence, by preprocessing $\mathcal{V}_{\operatorname{Pr}}(\mathcal{P})$ for point-location queries, for a query point $q$, we can report all $t$ quantification probabilities that are positive in $O(\log N+t)$ time.

THEOREM 4.2. Let $\mathcal{P}$ be a set of $n$ uncertain points in $\mathbb{R}^{2}$, each with a discrete distribution of size at most $k$; set $N=n k$. $\mathcal{P}$ can be preprocessed in time $O\left(N^{4} \log N\right)$ into a data structure of size $O\left(N^{4}\right)$ that can report all t positive quantification probabilities of a query point in time $O(\log N+t)$. 


\subsection{A Monte-Carlo Algorithm}

In this section, we describe a simple Monte-Carlo approach to build a data structure for quickly computing $\widehat{\pi}_{i}(q)$ for all $P_{i}$ for any query point $q$, which approximates the quantification probability $\pi_{i}(q)$. For a fixed value $s$, to be specified later, the preprocessing step works in $s$ rounds. In the $j$ th round, the algorithm creates a sample $R_{j}=\left\{r_{j 1}, r_{j 2}, \ldots, r_{j n}\right\} \subseteq \mathbb{R}^{2}$ by choosing each $r_{j i}$ using the distribution of $P_{i}$. For each $j \in\{1, \ldots, s\}$, we construct the Voronoi diagram $\operatorname{Vor}\left(R_{j}\right)$ in $O(n \log n)$ time and preprocess it for point-location queries in additional $O(n \log n)$ time.

To estimate quantification probabilities of a query $q$, we initialize a counter $c_{i}=0$ for each point $P_{i}$. For each $j \in\{1, \ldots, s\}$, we find the point $r_{j i} \in R_{j}$ whose cell in $\operatorname{Vor}\left(R_{j}\right)$ contains the query point $q$, and increment $c_{i}$ by 1 . Finally, we estimate $\widehat{\pi}_{i}(q)=c_{i} / s$. Note that at most $s$ distinct $c_{i}$ s have nonzero values, so we can implicitly set the remaining $\widehat{\pi}_{i}(q)$ s to 0 .

Discrete Case. If each $P_{i} \in \mathcal{P}$ has a discrete distribution of size $k$, then this algorithm can be implemented very efficiently. Each $r_{j i}$ can be selected in $O(\log k)$ time after preprocessing each $P_{i}$, in $O(k)$ time, into a balanced binary tree [Motwani and Raghavan 1995]. Thus, total preprocessing takes $O(s(n(\log n+\log k))+n k)=O(n k+s n \log (n k))$ time and $O(s n)$ space, and each query takes $O(s \log n)$ time.

It remains to determine the value of $s$ so that $\left|\pi_{i}(q)-\widehat{\pi}_{i}(q)\right| \leq \varepsilon$ for all $P_{i}$ and all queries $q$, with probability at least $1-\delta$. For fixed $q, P_{i}$, and instantiation $R_{j}$, let $\mathrm{X}_{j i}$ be the random indicator variable, which is 1 if $r_{j i}$ is the NN of $q$ and 0 otherwise. Since $\mathrm{E}\left[\mathrm{X}_{j i}\right]=\pi_{i}(q)$ and $\mathrm{X}_{i} \in\{0,1\}$, applying the Chernoff-Hoeffding bound [Motwani and Raghavan 1995] to

$$
\widehat{\pi}_{i}(q)=\frac{c_{i}}{s}=\frac{1}{s} \sum_{j=1}^{s} \mathrm{X}_{j i},
$$

we obtain that

$$
\operatorname{Pr}\left[\left|\widehat{\pi}_{i}(q)-\pi_{i}(q)\right| \geq \varepsilon\right] \leq 2 \exp \left(-2 \varepsilon^{2} s\right) .
$$

For each cell of $\mathcal{V}_{\operatorname{Pr}}(\mathcal{P})$, we choose one point, and let $Q$ be the resulting set of points. If $\left|\widehat{\pi}_{i}(q)-\pi_{i}(q)\right| \leq \varepsilon$ for every point $q \in Q$, then $\left|\widehat{\pi}_{i}(q)-\pi_{i}(q)\right| \leq \varepsilon$ for every point $q \in \mathbb{R}^{2}$. Since there are $n$ different values of $i$, by applying the union bound to Equation (6), the probability that there exist a point $q \in \mathbb{R}^{2}$ and an index $i \in\{1, \ldots, n\}$ with $\left|\widehat{\pi}_{i}(q)-\pi_{i}(q)\right| \geq$ $\varepsilon$ is at most $2 n|Q| \exp \left(-2 \varepsilon^{2} s\right)$. Hence, by setting

$$
s=\frac{1}{2 \varepsilon^{2}} \ln \frac{2 n|Q|}{\delta},
$$

$\left|\widehat{\pi}_{i}(q)-\pi_{i}(q)\right| \leq \varepsilon$ for all $q \in \mathbb{R}^{2}$ and for all $i \in\{1, \ldots, n\}$, with probability at least $1-\delta$. By Lemma 4.1, $|Q|=O\left(n^{4} k^{4}\right)$, so we obtain the following result.

THeOREM 4.3. Let $\mathcal{P}$ be a set of $n$ uncertain points in $\mathbb{R}^{2}$, each with a discrete distribution of size $k$, and let $\varepsilon, \delta \in(0,1)$ be two parameters. $\mathcal{P}$ can be preprocessed, in

$$
O\left(n k+\left(n / \varepsilon^{2}\right) \log (n k) \log (n k / \delta)\right)
$$

time, into a data structure of size $O\left(\left(n / \varepsilon^{2}\right) \log (n k / \delta)\right)$, which computes, for any query point $q \in \mathbb{R}^{2}$, in $O\left(\left(1 / \varepsilon^{2}\right) \log (n k / \delta) \log n\right)$ time, a value $\widehat{\pi}_{i}(q)$ for every $P_{i}$ such that $\mid \pi_{i}(q)-$ $\widehat{\pi}_{i}(q) \mid \leq \varepsilon$ for all $i$ with probability at least $1-\delta$.

Continuous Case. There are two technical issues in extending this technique and analysis to continuous distributions. First, how we instantiate a certain point $r_{i}$ from 
each $P_{i}$. Herein we assume the representation of the pdf is such that this can be done in constant time for each $P_{i}$.

Second, we need to bound the number of distinct queries that need to be considered to apply the union bound as we did earlier. Since $\pi_{i}(q)$ may vary continuously with the query location, unlike the discrete case, we cannot hope for a bounded number of distinct results. However, we just need to define a finite set $\bar{Q}$ of query points so that for any point $q \in \mathbb{R}^{2}$, there is a point $q^{\prime} \in \bar{Q}$ such that $\max _{i}\left|\pi_{i}(q)-\pi_{i}\left(q^{\prime}\right)\right| \leq \varepsilon / 2$. Then, we can choose $s$ large enough so that it permits at most $\varepsilon / 2$ error on each query in $\bar{Q}$. Specifically, choosing $s=O\left(\left(1 / \varepsilon^{2}\right) \log (n|\bar{Q}| / \delta)\right)$ is sufficient, so all that remains is to bound $|\bar{Q}|$.

To choose $\bar{Q}$, we show that each pdf of $P_{i}$ can be approximated with a discrete distribution of size $O\left(\left(n^{2} / \varepsilon^{2}\right) \log (n / \delta)\right)$, and then reduce the problem to the discrete case.

For parameters $\alpha>0$ and $\delta^{\prime} \in(0,1)$, set

$$
k(\alpha)=\frac{c}{\alpha^{2}} \log \frac{1}{\delta^{\prime}},
$$

where $c$ is a constant. For each $i \in\{1, \ldots, n\}$, we choose a random sample $\bar{P}_{i} \subset P_{i}$ of size $k(\alpha)$, according to the distribution defined by the location pdf $f_{i}$ of $P_{i}$. We regard $\bar{P}_{i}$ as an uncertain point with uniform location probability. Set $\overline{\mathcal{P}}=\left\{\bar{P}_{1}, \ldots, \bar{P}_{n}\right\}$.

For a point $q \in \mathbb{R}^{2}$, let $\bar{G}_{q, i}$ denote the cdf of the distance between $q$ and $\bar{P}_{i}$, that is, $\bar{G}_{q, i}(r)=\operatorname{Pr}\left[d\left(q, \bar{P}_{i}\right) \leq r\right]$, or equivalently, it is the probability of $\bar{P}_{i}$ lying in the disk of radius $r$ centered at $q$. A well-known result in the theory of random sampling [Li et al. 2001; Vapnik and Chervonenkis 1971] implies that for all $q \in \mathbb{R}^{2}$ and $r \geq 0$,

$$
\left|G_{q, i}(r)-\bar{G}_{q, i}(r)\right| \leq \alpha
$$

with probability at least $1-\delta^{\prime}$, provided that the constant $c$ in $k(\alpha)$ is chosen sufficiently large.

Let $\bar{\pi}_{i}(q)$ denote the probability of $\bar{P}_{i}$ being the NN of $q$ in $\overline{\mathcal{P}}$. We prove the following:

Lemma 4.4. For any $q \in \mathbb{R}^{2}$ and for any fixed $i \in\{1, \ldots, n\}$,

$$
\left|\pi_{i}(q)-\bar{\pi}_{i}(q)\right| \leq \alpha n,
$$

with probability at least $1-\delta^{\prime}$.

Proof. Recall that by Equation (1),

$$
\pi_{i}(q)=\int_{0}^{\infty} g_{q, i}(r) \prod_{j \neq i}\left(1-G_{q, j}(r)\right) \mathrm{d} r .
$$

Using Equation (7) and the fact that $G_{q, j}(r), \bar{G}_{q, j}(r) \in[0,1]$ for all $j$, we obtain

$$
\pi_{i}(q) \leq \int_{0}^{\infty} g_{q, i}(r) \prod_{j \neq i}\left(1-\bar{G}_{q, j}(r)\right) \mathrm{d} r+(n-1) \alpha .
$$

Note that $\prod_{j \neq i}\left(1-\bar{G}_{q, j}(r)\right)$ is the probability that the closest point of $q$ in $\overline{\mathcal{P}} \backslash\left\{\bar{P}_{i}\right\}$ is at least distance $r$ away from $q$. Let $h_{q, i}$ be the pdf of the distance between $q$ and its closest point in $\overline{\mathcal{P}} \backslash\left\{\bar{P}_{i}\right\}$. Then

$$
\prod_{j \neq i}\left(1-\bar{G}_{q, j}(r)\right)=\int_{r}^{\infty} h_{q, i}(\theta) \mathrm{d} \theta .
$$


Therefore,

$$
\pi_{i}(q) \leq \int_{0}^{\infty} \int_{r}^{\infty} g_{q, i}(r) h_{q, i}(\theta) \mathrm{d} \theta \mathrm{d} r+(n-1) \alpha .
$$

By reversing the order of integration, we obtain

$$
\begin{aligned}
\pi_{i}(q) & \leq \int_{0}^{\infty} \int_{0}^{\theta} h_{q, i}(\theta) g_{q, i}(r) \mathrm{d} r \mathrm{~d} \theta+(n-1) \alpha \\
& =\int_{0}^{\infty} h_{q, i}(\theta) G_{q, i}(\theta) \mathrm{d} \theta+(n-1) \alpha \\
& \leq \int_{0}^{\infty} h_{q, i}(\theta)\left(\bar{G}_{q, i}(\theta)+\alpha\right) \mathrm{d} \theta+(n-1) \alpha \\
& (\text { using Equation }(7)) \\
& =\int_{0}^{\infty} h_{q, i}(\theta) \bar{G}_{q, i}(\theta) \mathrm{d} \theta+n \alpha \\
& =\bar{\pi}_{i}(q)+n \alpha .
\end{aligned}
$$

A similar argument shows that $\pi_{i}(q) \geq \bar{\pi}_{i}(q)-n \alpha$. This completes the proof of the lemma.

Thus, by setting $\alpha=\varepsilon /(2 n)$, a random sample $\bar{P}_{i}$ of size $O\left(\left(n^{2} / \varepsilon^{2}\right) \log (n / \delta)\right)$ from each $P_{i}$ ensures that

$$
\left|\pi_{i}(q)-\bar{\pi}_{i}(q)\right| \leq \varepsilon / 2
$$

for all queries. By choosing $\delta^{\prime}=\delta /(2 n)$, Equation (8) holds for all $i \in\{1, \ldots, n\}$ with probability at least $1-\delta / 2$.

We consider $\mathcal{V}_{\operatorname{Pr}}(\overline{\mathcal{P}})$, choose one point from each of its cells, and set $\bar{Q}$ to be the resulting set of points. For a point $q \in \mathbb{R}^{2}$, let $\bar{q} \in \bar{Q}$ be the representative point of the cell of $\mathcal{V}_{\operatorname{Pr}}(\overline{\mathcal{P}})$ that contains $q$. Then, $\left|\pi_{i}(q)-\bar{\pi}_{i}(\bar{q})\right|<\varepsilon / 2$ for all points $q \in \mathbb{R}^{2}$ and $i \in\{1, \ldots, n\}$, with probability at least $1-\delta / 2$.

Now, applying the analysis for the discrete case to the point set $\overline{\mathcal{P}}$, if we choose

$$
s=O\left(\frac{1}{\varepsilon^{2}} \log \frac{n|\bar{Q}|}{\delta}\right),
$$

then $\left|\bar{\pi}_{i}(q)-\widehat{\pi}_{i}(q)\right|<\varepsilon$ for all points $q \in \mathbb{R}^{2}$ and for all $i \in\{1, \ldots, n\}$ with probability at least $1-\delta / 2$. Since

$$
\left|\bar{P}_{i}\right|=k\left(\frac{\varepsilon}{2 n}\right)=O\left(\frac{n^{2}}{\varepsilon^{2}} \log \frac{n}{\delta}\right)
$$

by Lemma 4.1 ,

$$
|\bar{Q}|=O\left(n^{4}\left(k\left(\frac{\varepsilon}{2 n}\right)\right)^{4}\right)=O\left(\frac{n^{12}}{\varepsilon^{8}} \log ^{4} \frac{n}{\delta}\right) .
$$

Putting everything together, we obtain the following.

TheOREM 4.5. Let $\mathcal{P}=\left\{P_{1}, \ldots, P_{n}\right\}$ be a set of $n$ uncertain points in $\mathbb{R}^{2}$ so a random instantiation of $P_{i}$ can be performed in $O(1)$ time, and let $\varepsilon, \delta \in(0,1)$ be two parameters. $\mathcal{P}$ can be preprocessed in $O\left(\left(n / \varepsilon^{2}\right) \log (n / \varepsilon \delta) \log n\right)$ time into a data structure of size $O\left(\left(n / \varepsilon^{2}\right) \log (n / \varepsilon \delta)\right)$ that computes for any query point $q \in \mathbb{R}^{2}$, in $O\left(\left(1 / \varepsilon^{2}\right) \log (n / \varepsilon \delta) \log n\right)$ 
time, a value $\widehat{\pi}_{i}(q)$ for every $P_{i}$ such that $\left|\pi_{i}(q)-\widehat{\pi}_{i}(q)\right| \leq \varepsilon$ for all $i$ with probability at least $1-\delta$.

\subsection{Spiral Search Algorithm}

If the distribution of each point in $\mathcal{P}$ is discrete, then there is an alternative approach to approximate the quantification probabilities for a given query $q$ : set a parameter $m>1$, choose the $m$ points of $S=\bigcup_{i=1}^{n} P_{i}$ that are closest to $q$, and use only these $m$ points to estimate $\pi_{i}(q)$ for each $P_{i}$. We show that this works for a small value of $m$ when, for each $P_{i}$, each location is approximately equally likely, but is not efficient if the location probabilities vary significantly.

Recall that $w_{i j}$ is the location probability of a point $p_{i j} \in P_{i}$. Set $S=\bigcup_{i=1}^{n} P_{i}$ to be the set of all possible locations of points in $\mathcal{P}$. We define the quantity

$$
\rho=\frac{\max _{i, j} w_{i j}}{\min _{i, j} w_{i j}}
$$

the ratio of the largest to the smallest location probability over all points of $S$, as the spread of location probabilities. Set

$$
m(\rho, \varepsilon)=\rho k \ln (1 / \varepsilon)+k-1 .
$$

Fix a query point $q \in \mathbb{R}^{2}$. Let $\bar{S} \subseteq S$ be the $m(\rho, \varepsilon)$ nearest neighbors of $q$ in $S$, $\bar{P}_{i}=\bar{S} \cap P_{i}$, and $\overline{\mathcal{P}}=\left\{\bar{P}_{1}, \ldots, \bar{P}_{n}\right\}$. Note that $\bar{w}_{i}=\sum_{p_{i, a} \in \bar{P}_{i}} w_{i, a}$ is not necessarily equal to 1 , so we cannot regard $\bar{P}_{i}$ as an uncertain point in our model, but still it will be useful to think of $\bar{P}_{i}$ as an uncertain point that does not exist with probability $1-\bar{w}_{i}$.

For a set $Y$ of points and another point $\xi \in \mathbb{R}^{2}$, let

$$
Y[\xi]=\{p \in Y \mid d(q, p) \leq d(q, \xi)\} .
$$

For a point $p:=p_{i, a} \in P_{i}$, the probability that $p$ is the NN of $q$ in $\mathcal{P}$, denoted by $\eta(p ; q)$, is

$$
\eta(p ; q)=w_{i, a} \prod_{j \neq i}\left(1-\sum_{p_{j, \ell} \in P_{j}[p]} w_{j, \ell}\right) .
$$

Moreover,

$$
\pi_{i}(q)=\sum_{p_{i, a} \in P_{i}} \eta\left(p_{i, a} ; q\right) .
$$

For each $i \leq n, q \in \mathbb{R}^{2}$, and $p_{i, a} \in \bar{P}_{i}$, we analogously define the quantities $\widehat{\eta}\left(p_{i, a} ; q\right)$ and $\widehat{\pi}_{i}(q)$ using Equation (10) and Equation (11) but replacing $P_{j}$ with $\bar{P}_{j}$ for every $j \in\{1, \ldots, n\}$. Intuitively, if $\overline{\mathcal{P}}$ were a family of uncertain points, then $\widehat{\pi}_{i}(q)$ would be the probability of $\bar{P}_{i}$ being the NN of $q$ in $\overline{\mathcal{P}}$.

Lemma 4.6. For all $i \in\{1, \ldots, n\}$,

$$
\widehat{\pi}_{i}(q) \leq \pi_{i}(q) \leq \widehat{\pi}_{i}(q)+\varepsilon .
$$

Proof. Fix a point $p \in P_{i}$. If $p \in \bar{P}_{i}$, then for all $j \neq i, \bar{P}_{j}[p]=P_{j}[p]$; therefore, by Equation $(10), \eta(p ; q)=\widehat{\eta}(p ; q)$. 
Hence, by Equation (11),

$$
\begin{aligned}
\pi_{i}(q) & =\sum_{p \in \bar{P}_{i}} \eta(p ; q)+\sum_{p \in P_{i} \backslash \bar{P}_{i}} \eta(p ; q) \\
& =\sum_{p \in \bar{P}_{i}} \widehat{\eta}(p ; q)+\sum_{p \in P_{i} \backslash \bar{P}_{i}} \eta(p ; q) \\
& =\widehat{\pi}_{i}(q)+\sum_{p \in P_{i} \backslash \bar{P}_{i}} \eta(p ; q) .
\end{aligned}
$$

Therefore, $\widehat{\pi}_{i}(q) \leq \pi_{i}(q)$. Next, we bound the second term in the right-hand side of Equation (12). Let $p \in P_{i} \backslash \bar{P}_{i}$. Set $x_{j}=\left|P_{j}[p]\right|$, for $j \neq i$, and $m^{\prime}=\sum_{j \neq i} x_{j}$. Since $P_{i} \backslash \bar{P}_{i} \neq \emptyset,\left|\bar{P}_{i}\right| \leq k-1$ and $m^{\prime}=|\bar{S}|-\left|\bar{P}_{i}\right| \geq \rho k \ln (1 / \varepsilon)$. Note that each $w_{j, a} \geq 1 / \rho k$. Therefore,

$$
\begin{aligned}
\eta(p ; q) & =w_{i, a} \prod_{j \neq i}\left(1-\sum_{p_{\ell} \in P_{j}[p]} w_{j, \ell}\right) \\
& \leq w_{i, a} \prod_{j \neq i}\left(1-\frac{x_{j}}{\rho k}\right) \leq w_{i, a} \prod_{j \neq i} \exp \left(-x_{j} / \rho k\right) \\
& =w_{i, a} \exp \left(-m^{\prime} / \rho k\right) \leq w_{i, a} \varepsilon .
\end{aligned}
$$

Consequently,

$$
\sum_{p \in P_{i} \backslash \bar{P}_{i}} \eta(p ; q) \leq \sum_{p \in P_{i} \backslash \bar{P}_{i}} \varepsilon w_{i, a} \leq \varepsilon .
$$

Plugging Equation (13) into Equation (12), we obtain $\pi_{i}(q) \leq \widehat{\pi}_{i}(q)+\varepsilon$, as claimed. This completes the proof of the lemma.

For any $i$, if $P_{i} \cap \bar{S}(q)=\varnothing$, then we can implicitly set $\widehat{\pi}_{i}(q)$ to 0 . Using the data structure by Afshani and Chan [2009], $S$ can be preprocessed in $O(N \log N)$ randomized expected time into a data structure of $O(N)$ size so that $m:=m(\rho, \varepsilon)$ nearest neighbors of a query point can be reported in $O(m+\log N)$ time. We thus obtain the following result.

THEOREM 4.7. Let $\mathcal{P}$ be a set of $n$ uncertain points in $\mathbb{R}^{2}$, each with a discrete distribution of size at most $k$; let $\rho$ be the spread of the location probabilities; and let $N=n k$. $\mathcal{P}$ can be preprocessed in $O(N \log N)$ expected time into a data structure of size $O(N)$, so that for a query point $q \in \mathbb{R}^{2}$ and a parameter $\varepsilon \in(0,1)$, it can compute, in time $O(\rho k \log (1 / \varepsilon)+\log N)$, values $\widehat{\pi}_{i}(q)$ for all $P_{i} \in \mathcal{P}$ such that $\left|\pi_{i}(q)-\widehat{\pi}_{i}(q)\right| \leq \varepsilon$ for all $i \in\{1, \ldots, n\}$.

Remarks. (i) This approach is not efficient when the spread of location probabilities is unbounded. In this case, one may have to retrieve $\Omega(n)$ points. Another approach may be to ignore points with weight smaller than $\varepsilon / k$, since even $k$ such weights from a single uncertain point $P_{i}$ cannot contribute more than $\varepsilon$ to $\pi_{i}(q)$. However, the union of all such points may distort other probabilities.

Consider the following example. Let $p_{1} \in P_{1} \in \mathcal{P}$ be the closest point to the query point $q$. Let $w\left(p_{1}\right)=3 \varepsilon$. Let the next $n / 2$ closest points $p_{3}, \ldots, p_{n / 2+2}$ be from different uncertain points $P_{3}, \ldots, P_{n / 2+2}$ and each have weights $w(p)=2 / n \ll \varepsilon / k$. Let the next closest point $p_{2} \in P_{2} \in \mathcal{P}$ have weight $w\left(p_{2}\right)=5 \varepsilon$. With probability $\pi_{p_{1}}(q)=3 \varepsilon$, the 
nearest neighbor is $p_{1}$. The probability that $p_{2}$ is the nearest neighbor is $\pi_{p_{2}}(q)=$ $(5 \varepsilon)(1-3 \varepsilon)(1-2 / n)^{n / 2}<(5 \varepsilon)(1-3 \varepsilon)(1 / e)<2 \varepsilon$. Thus, $p_{1}$ is more likely to be the nearest neighbor than $p_{2}$. However, if we ignore points $p_{3}, \ldots, p_{n / 2+2}$ because they have small weights, then we calculate that $p_{2}$ has probability $\widehat{\pi}_{p_{2}}(q)=(1-3 \varepsilon)(5 \varepsilon)>4 \varepsilon$ for being the nearest neighbor (assuming that $\varepsilon$ is small enough). So $\pi_{2}(q)$ will be off by more than $2 \varepsilon$ and it would incorrectly appear that $p_{2}$ is more likely to be the nearest neighbor than $p_{1}$.

(ii) Though the data structure by Afshani and Chan [2009] is optimal theoretically, it is too complex to be implemented. Instead, one may use the order- $m$ Voronoi diagram to retrieve the $m$ closest points (in unsorted order) to $q$. This would yield a data structure with $O(m(n k-m))$ space and $O\left(m(n k-m) \log (n k)+n k \log ^{3}(n k)\right)$ expected preprocessing time [Agarwal et al. 1998], while preserving the query time $O(\log (n k)+m)$, where $m=O(\rho k \log (\rho / \varepsilon))$. Alternatively, one may use quad-trees and a branch-and-bound algorithm to retrieve $m$ points of $S$ closest to $q$ [Har-Peled 2011].

\section{CONCLUSION}

In this article, we investigated NN queries in a probabilistic framework in which the location of each input point is specified as a probability distribution function. We presented efficient methods for returning all points with nonzero probability of being the nearest neighbor, estimating the quantification probabilities and using it for threshold NN queries. We conclude by mentioning two open problems:

(i) The lower-bound constructions for the complexity of $\mathcal{V}_{\neq 0}(\mathcal{P})$ are created very carefully, and these configurations are unlikely to occur in practice. A natural question is to characterize the sets of uncertain points for which the complexity of $\mathcal{V}_{\neq 0}(\mathcal{P})$ is near linear.

(ii) Are there simple and practical linear-size data structures for answering $\mathrm{NN}_{\neq 0}$ queries in sublinear time?

(iii) Can we extend the spiral search method to continuous distributions (at least for some simple, well-behaved distributions, such as Gaussian), so that the query time is always sublinear?

\section{REFERENCES}

P. Afshani and T. M. Chan. 2009. Optimal halfspace range reporting in three dimensions. In Proc. 20th ACM-SIAM Sympos. Discrete Algs. 180-186.

P. K. Agarwal. 2016. Range searching. In Handbook of Discrete and Computational Geometry (3rd ed.), J. E. Goodman, J. O'Rourke, and C. Toth (Eds.). CRC Press, Chapter 41, to appear.

P. K. Agarwal, M. de Berg, J. Matoušek, and O. Schwarzkopf. 1998. Constructing levels in arrangements and higher order Voronoi diagrams. SIAM J. Comput. 27 (1998), 654-667.

P. K. Agarwal, A. Efrat, S. Sankararaman, and W. Zhang. 2012. Nearest-neighbor searching under uncertainty. In Proc. 31st ACM Sympos. Principles Database Syst. 225-236.

P. K. Agarwal and M. Sharir. 2000. Arrangements and their applications. In Handbook of Computational Geometry, J.-R. Sack and J. Urrutia (Eds.). North-Holland Publishing Co., Amsterdam, 49-119.

C. C. Aggarwal. 2009. Managing and Mining Uncertain Data. Springer-Verlag.

P. F. Ash and E. D. Bolker. 1986. Generalized Dirichlet tessellations. Geometriae Dedicata 20, 2 (1986), 209-243.

T. Bernecker, T. Emrich, H.-P. Kriegel, N. Mamoulis, M. Renz, and A. Zuefle. 2011. A novel probabilistic pruning approach to speed up similarity queries in uncertain databases. In Proc. 27th IEEE Int. Conf. Data Eng. 339-350.

G. Beskales, M. A. Soliman, and I. F. IIyas. 2008. Efficient search for the top-k probable nearest neighbors in uncertain databases. Proc. VLDB Endow. 1, 1 (2008), 326-339.

R. Cheng, J. Chen, M. Mokbel, and C. Chow. 2008. Probabilistic verifiers: Evaluating constrained nearestneighbor queries over uncertain data. In Proc. 24th IEEE Int. Conf. Data Eng. 973-982. 
R. Cheng, L. Chen, J. Chen, and X. Xie. 2009. Evaluating probability threshold $k$-nearest-neighbor queries over uncertain data. In Proc. 12th Int. Conf. Ext. Database Tech. 672-683.

R. Cheng, D. V. Kalashnikov, and S. Prabhakar. 2004. Querying imprecise data in moving object environments. IEEE Trans. Know. Data Eng. 16, 9 (2004), 1112-1127.

R. Cheng, X. Xie, M. L. Yiu, J. Chen, and L. Sun. 2010. UV-diagram: A Voronoi diagram for uncertain data. In Proc. 26th IEEE Int. Conf. Data Eng. 796-807.

X. Dai, M. L. Yiu, N. Mamoulis, Y. Tao, and M. Vaitis. 2005. Probabilistic spatial queries on existentially uncertain data. In Proc. 9th Int. Sympos. Spatial Temporal Databases. 400-417.

N. N. Dalvi, C. Ré, and D. Suciu. 2009. Probabilistic databases: Diamonds in the dirt. Commun. ACM 52, 7 (2009), 86-94.

M. de Berg, O. Cheong, M. van Kreveld, and M. H. Overmars. 2008. Computational Geometry: Algorithms and Applications (3rd ed.). Springer-Verlag.

J. R. Driscoll, N. Sarnak, D. D. Sleator, and R. E. Tarjan. 1989. Making data structures persistent. J. Comput. Syst. Sci. 38 (1989), 86-124.

S. Har-Peled. 2011. Geometric Approximation Algorithms. Mathematical Surveys and Monographs, Vol. 173. American Mathematical Society.

J. Jestes, G. Cormode, F. Li, and K. Yi. 2011. Semantics of ranking queries for probabilistic data. IEEE Trans. Know. Data Eng. 23, 12 (2011), 1903-1917.

P. Kamousi, T. M. Chan, and S. Suri. 2014. Closest pair and the post office problem for stochastic points. Comput. Geom. Theory Appl. 47, 2 (2014), 214-223.

H. Kaplan, W. Mulzer, L. Roditty, P. Seiferth, and M. Sharir. 2016. Dynamic planar Voronoi diagrams for general distance functions and their algorithmic applications. CoRR abs/1604.03654 (2016).

H.-P. Kriegel, P. Kunath, and M. Renz. 2007. Probabilistic nearest-neighbor query on uncertain objects. In Proc. 12th Int. Conf. Database Sys. Adv. App. 337-348.

Y. Li, P. M. Long, and A. Srinivasan. 2001. Improved bounds on the sample complexity of learning. J. Comput. Syst. Sci. 62, 3 (2001), 516-527.

V. Ljosa and A. K. Singh. 2007. APLA: Indexing arbitrary probability distributions. In Proc. 23rd IEEE Int. Conf. Data Eng. 946-955.

R. Motwani and P. Raghavan. 1995. Randomized Algorithms. Cambridge University Press, Cambridge, UK.

J. Sember and W. Evans. 2008. Guaranteed Voronoi diagrams of uncertain sites. In Proc. 20th Canad. Conf. Comput. Geom. 207-210.

M. Sharir and P. K. Agarwal. 1995. Davenport-Schinzel Sequences and Their Geometric Applications. Cambridge University Press.

V. N. Vapnik and A. Y. Chervonenkis. 1971. On the uniform convergence of relative frequencies of events to their probabilities. Theory Probab. Appl. 16 (1971), 264-280.

S. M. Yuen, Y. Tao, X. Xiao, J. Pei, and D. Zhang. 2010. Superseding nearest neighbor search on uncertain spatial databases. IEEE Trans. Know. Data Eng. 22, 7 (2010), 1041-1055.

P. Zhang, R. Cheng, N. Mamoulis, M. Renz, A. Zufile, Y. Tang, and T. Emrich. 2013. Voronoi-based nearest neighbor search for multi-dimensional uncertain databases. In Proc. 29th IEEE Int. Conf. Data Eng. 158-169.

Received February 2015; revised May 2016; accepted June 2016 